\title{
II: ANORDNUNG DER PERIKOPEN
}

Die folgende Auflistung gibt einen Überblick über die Perikopenanordnung in den vier Handschriften. In der ersten Spalte steht die Nummer, unter der die Perikope bzw. der Perikopenverweis jeweils im Editionsteil aufgeführt ist. Kursive Nummern zeigen an, daß die Leithandschrift Fr hier ausfallt. Damit man wie in Spalte 4 (Fr) auch in den Spalten 5-7 (Br / N1 / N2) die Reihenfolge in allen Handschriften vor Augen hat, sind außerhalb der Nummernfolge Verweise auf Umstellungen eingefügt. Sie sind an den mit runden Klammern umschlossenen Nummern zu erkennen. In Spalte 2 steht die moderne Tagesbezeichnung, die sich auf die Angaben der Leithandschrift Fr bezieht. Der zugrundeliegende handschriftliche Wortlaut kann im Editionsteil leicht aufgefunden werden. Wo eine Datumsangabe erforderlich ist wie etwa bei den Heiligenfesten, ist sie in eckigen Klammern hinzugefügt. Der Normtag (Spalte 3) ist der Übersicht bei PAUL PIETSCH${ }^{1}$ entnommen und bezieht sich auf die jeweilige Perikope im Bremer Evangelistar. Ist der Normtag mit dem Tag in der Handschrift (Spalte 2) identisch, dann entfallt ein Eintrag. Ist der Normtag nur im ältesten gedruckten Plenar nachgewiesen, so steht (A), ist er nur in dem von Adam Petri gedruckten niederdeutschen Plenar nachgewiesen, so steht $(\mathrm{N})$ in Spale 3. In Spalte 4 steht die jeweilige Evangelienstelle. Nur bei Auslassungen von mehreren Versen werden diese hier und gegebenenfalls in den folgenden drei Spalten angeben. In der jeweils ersten Zeile von Spalte 4-7 zeigt ein Gleichheitszeichen an, daß die entsprechende Perikope auch in dieser Handschrift bezeugt ist. Fehlt sie, so wird dies durch $\bigcirc$ angezeigt. In der zweiten Zeile ist bei einer abweichenden Tagesbezeichnung diese angegeben; fehlt sie bei einer Perikope, so wird dies durch $\circ$ angezeigt. Die in der Handschrift N1 von späterer Hand an den Rändern eingetragenen Tagesbezeichnungen werden in geschweiften Klammern in den Anmerkungen mitgeteilt. Hinsichtlich der Umsetzung der mittelalterlichen in neuzeitliche Tagesbezeichnungen sei auf das Standardwerk von HERMANN GROTEFEND ${ }^{2}$ verwiesen.

1 Ewangely und Epistel Teutsch. Die gedruckten hochdeutschen Perikopenbücher (Plenarien) 1473-1523, Göttingen 1927, S. 59-114.

2 Zeitrechnung des deutschen Mittelalters und der Neuzeit, 2 Bde, Hannover 1891-98. 


\section{TEMPORALE}

\begin{tabular}{|c|c|c|c|c|c|c|}
\hline Nr. & Tag in Hs. & Normtag & Fr & $\mathbf{B r}$ & N1 & N2 \\
\hline 1 & $\begin{array}{l}\text { 1. Adventssonn- } \\
\text { tag }\end{array}$ & 2. Adventssonntag & Lk 21, 25-33 & $\begin{array}{l}= \\
0\end{array}$ & $=$ & $\circ$ \\
\hline 2 & Dienstag & 3. Adventssonntag & Mt 11, 2-10 & $=$ & $\begin{array}{l}= \\
\text { Donnerstag }\end{array}$ & $\circ$ \\
\hline 3 & $\begin{array}{l}\text { Quatembermitt- } \\
\text { woch }\end{array}$ & $\begin{array}{l}\text { [= Mi. n. 3. Ad- } \\
\text { ventssonntag] }\end{array}$ & $\begin{array}{l}\text { Lk 1, 26-38 } \\
\text { (vgl. 169) }\end{array}$ & $\begin{array}{l}= \\
0 \\
\text { (vgl. 169) }\end{array}$ & $\begin{array}{l}= \\
\text { Quatember- } \\
\text { mittwoch } \\
\text { (vgl. 169) }\end{array}$ & $\circ$ \\
\hline 4 & & & $\circ$ & $\begin{array}{l}\text { Lk 1, 26-38 } \\
0 \\
\text { (Nachtrag in } \\
\text { Hs nach 223) }\end{array}$ & $\circ$ & $\circ$ \\
\hline 5 & & & $\circ$ & $\circ$ & $\circ$ & $\begin{array}{l}\text { Lk 1, 26-38 } \\
\text { Mittwoch } \\
\text { (in Hs bei 169) }\end{array}$ \\
\hline 6 & Freitag & $\begin{array}{l}\text { [= Fr. n. 3. Ad- } \\
\text { ventssonntag } \\
\text { (Quatember)] }\end{array}$ & $\begin{array}{l}\text { Lk 1, 39-47 } \\
\text { (vgl. 170) }\end{array}$ & $\begin{array}{l}= \\
0 \\
\text { (vgl. 170) }\end{array}$ & $\begin{array}{l}= \\
\text { (vgl. 170) }\end{array}$ & $\circ$ \\
\hline 7 & & & $\circ$ & $\circ$ & $\circ$ & $\begin{array}{l}\text { Lk 1, 39-47 } \\
\text { (in Hs bei 170) }\end{array}$ \\
\hline 8 & $\begin{array}{l}\text { 4. Adventssonn- } \\
\text { tag }\end{array}$ & & Jo $1,19-28$ & $=$ & $={ }^{3}$ & $\circ$ \\
\hline 9 & Samstag & $\begin{array}{l}\text { Sa. n. 3. Advents- } \\
\text { sonntag } \\
\text { [Quatember] }\end{array}$ & Lk 3, 1-6 & $\begin{array}{l}= \\
\text { Freitag }\end{array}$ & $\begin{array}{l}= \\
\text { Freitag getilgt }\end{array}$ & $\circ$ \\
\hline
\end{tabular}

10 Weihnachtsabend [24.12.]

Mt 1, 18-21=

$=$

11 Weihnachten

[25.12]

1. Messe

Lk 2, 1-14

3 \{Verweis auf 9 als Quatembersamstag\} 


\begin{tabular}{|c|c|c|c|c|c|}
\hline Nr. & Tag in Hs. & $\mathbf{F r}$ & $\mathrm{Br}$ & N1 & $\mathbf{N 2}$ \\
\hline 12 & $\begin{array}{l}\text { Weihnachten } \\
\text { 2. Messe }\end{array}$ & Lk 2, 15-20 & $=$ & $=$ & $=$ \\
\hline 13 & 3. Messe & Jo $1,1-14$ & $=$ & $=$ & $=$ \\
\hline 14 & $\begin{array}{l}\text { Stephanus } \\
\text { [26.12.] }\end{array}$ & Mt 23, 34-39 & $=$ & $=$ & $=$ \\
\hline 15 & $\begin{array}{l}\text { Johannes } \\
\text { [27.12.] }\end{array}$ & Jo 21, 19-24 & $=$ & $=$ & $=$ \\
\hline 16 & $\begin{array}{l}\text { Unschuldige } \\
\text { Kinder [28.12.] }\end{array}$ & Mt 2, 13-18 & $=$ & $=$ & $=$ \\
\hline 17 & Neujahr [1.1.] & Lk 2, 21 & $=$ & $=$ & $=$ \\
\hline 18 & $\begin{array}{l}\text { Sonntag n. Neu- } \\
\text { jahr }\end{array}$ & o & $\circ$ & Lk 2, 33-40 4 & o \\
\hline 19 & Dreikønig [6.1.] & Mt 2, 1-12 & $=$ & $\begin{array}{l}= \\
\text { Vorabend von } \\
\text { Dreikönig }\end{array}$ & $=$ \\
\hline
\end{tabular}

\begin{tabular}{|c|c|c|c|c|c|}
\hline 20 & $\begin{array}{l}\text { 1. So. n. Dreiks- } \\
\text { nig }\end{array}$ & 0 & Lk 2, 42-52 & $\begin{array}{l}= \\
\text { Dreikōnig }\end{array}$ & $\begin{array}{l}= \\
\text { Mittwoch }\end{array}$ \\
\hline 21 & Donnerstag & Okaav v. Dreikonig o & $\circ$ & $\begin{array}{l}= \\
\text { Mittwoch }\end{array}$ & Mt 3, 13-17 \\
\hline
\end{tabular}

\begin{tabular}{|c|c|c|c|c|c|c|}
\hline 22 & $\begin{array}{l}\text { Oktav von Drei- } \\
\text { könig }\end{array}$ & $\begin{array}{l}\text { Fr. n. 1. So. n. } \\
\text { Dreikönig (N) }\end{array}$ & $\begin{array}{l}\text { Mt 4, 12-17 } \\
\text { (12-14 fehlt) }\end{array}$ & $\begin{array}{l}= \\
\text { Mittwoch }\end{array}$ & $\begin{array}{l}= \\
\text { Freitag }\end{array}$ & $\begin{array}{l}= \\
\text { Freitag }\end{array}$ \\
\hline 23 & - & Fronleichnam & $\circ$ & $\begin{array}{l}\text { Jo 6, } 54-59 \\
\text { (in Hs in zwei } \\
\text { Teilen noch- } \\
\text { mals nach } 91 \\
\text { und 93) }\end{array}$ & $\circ$ & $\circ$ \\
\hline 24 & $\begin{array}{l}\text { 1. So. n. Dreiks- } \\
\text { nig }\end{array}$ & $\begin{array}{l}\text { Vorabend v. Drei- } \\
\text { konig }\end{array}$ & $\circ$ & Mt 2, 19-23 & $\circ$ & $\begin{array}{l}= \\
\text { (in Hs noch- } \\
\text { mals nach 19) }\end{array}$ \\
\hline
\end{tabular}

4 Sonntag in der Festwoche von Weihnachten\} 


\begin{tabular}{|c|c|c|c|c|c|c|}
\hline Nr. & Tag in Hs. & Normtag & $\mathbf{F r}$ & $\mathbf{B r}$ & N1 & $\mathbf{N} 2$ \\
\hline 25 & $\begin{array}{l}\text { Freitag n. 1. So } \\
\text { n. Dreikönig }\end{array}$ & $\begin{array}{l}\text { Mi. n. 1. So. n. } \\
\text { Dreikőnig (N) }\end{array}$ & Jo 1, 29-34 & $\begin{array}{l}= \\
\text { Freitag }\end{array}$ & $\begin{array}{l}= \\
\text { 1. So. n. } \\
\text { Dreikōnig } 5\end{array}$ & $\begin{array}{l}= \\
0\end{array}$ \\
\hline 26 & $\begin{array}{l}\text { 2. So. n. Dreikö- } \\
\text { nig }\end{array}$ & & Jo 2, 1-11 & $=$ & $\begin{array}{l}= \\
\text { 1. So. n. } \\
\text { Weihnachten }\end{array}$ & $\begin{array}{l}= \\
\text { 1. So. n. } \\
\text { Dreikönig }\end{array}$ \\
\hline 27 & $\begin{array}{l}\text { Mittwoch n. } 1 . \\
\text { So. n. Dreikönig }\end{array}$ & $\begin{array}{l}\text { Fr. n. 2. So. n. } \\
\text { Dreikönig (N) }\end{array}$ & Lk 4, 14-22 & $\begin{array}{l}= \\
\text { Mittwoch }\end{array}$ & $\begin{array}{l}= \\
\text { Mittwoch }\end{array}$ & $\begin{array}{l}= \\
\text { Mittwoch }\end{array}$ \\
\hline 28 & $\begin{array}{l}\text { Freitag n. } 2 . \text { So. } \\
\text { n. Dreikōnig }\end{array}$ & $\begin{array}{l}\text { Mi. n. 2. So. n. } \\
\text { Dreikōnig (N) }\end{array}$ & Lk 4, 31-37 & $\begin{array}{l}= \\
\text { Freitag }\end{array}$ & $\begin{array}{l}= \\
\text { Freitag }\end{array}$ & $\begin{array}{l}= \\
\text { Freitag }\end{array}$ \\
\hline 29 & - & $\begin{array}{l}\text { 3. So. n. Dreikō- } \\
\text { nig }\end{array}$ & $\underset{\circ}{\text { Mt 8, 1-13 }}$ & $\begin{array}{l}= \\
\text { 3. So. } \mathrm{n} . \\
\text { Dreikönig }\end{array}$ & $\begin{array}{l}= \\
\text { 1. So. n. } \\
\text { Dreikōnig } \\
\text { (5-13 fehlt; } \\
\text { vgl. aber 45) }\end{array}$ & $\begin{array}{l}= \\
\text { 3. So. n. } \\
\text { Dreikōnig }\end{array}$ \\
\hline 30 & $\begin{array}{l}\text { Mittwoch n. } 1 . \\
\text { So. n. Dreikönig }\end{array}$ & $\begin{array}{l}\text { Mi. n. 3. So. n. } \\
\text { Dreikönig (N) }\end{array}$ & $\begin{array}{l}\text { Mt 4, 23-25 } \\
\text { Freitag getilgt }\end{array}$ & $\begin{array}{l}= \\
\text { Mittwoch }\end{array}$ & $\begin{array}{l}= \\
\text { Mittwoch }\end{array}$ & $\begin{array}{l}= \\
\text { Mittwoch }\end{array}$ \\
\hline 31 & $\begin{array}{l}\text { Freitag n. 1. So. } \\
\text { n. Dreikōnig }\end{array}$ & $\begin{array}{l}\text { Fr. n. 3. So. n. } \\
\text { Dreikōnig (N) }\end{array}$ & Mk 3, 6-15 & $\begin{array}{l}= \\
\text { Freitag }\end{array}$ & $\begin{array}{l}= \\
\text { Freitag }\end{array}$ & $\begin{array}{l}= \\
\text { Freitag }\end{array}$ \\
\hline 32 & $\begin{array}{l}\text { 4. So. n. Dreikö- } \\
\text { nig }\end{array}$ & & Mt 8, 23-27 & $\begin{array}{l}= \\
\text { 5. So. n. } \\
\text { Dreikönig }\end{array}$ & $\begin{array}{l}= \\
\text { 3. So. n. } \\
\text { Dreikőnig }\end{array}$ & $\begin{array}{l}= \\
\text { 4. So. }\end{array}$ \\
\hline 33 & $\begin{array}{l}\text { Mittwoch n. } 4 . \\
\text { So. n. Dreikönig }\end{array}$ & - & Mk 6, 1-6 & $\begin{array}{l}= \\
\text { Mittwoch }\end{array}$ & $\begin{array}{l}= \\
\text { Mittwoch }\end{array}$ & $\begin{array}{l}= \\
\text { Mittwoch }\end{array}$ \\
\hline 34 & $\begin{array}{l}\text { Freitag n. } 4 \text {. So. } \\
\text { n. Dreikōnig }\end{array}$ & $\begin{array}{l}\text { Mi. n. 4. So. n. } \\
\text { Dreikōnig (N) }\end{array}$ & Lk 9, 57-62 & $\begin{array}{l}= \\
\text { Freitag }\end{array}$ & $\begin{array}{l}= \\
\text { Freitag }\end{array}$ & $\begin{array}{l}= \\
\text { Freitag }\end{array}$ \\
\hline 35 & $\begin{array}{l}\text { 5. So. n. Dreikd- } \\
\text { nig }\end{array}$ & -6 & Mt $11,25-30$ & $\begin{array}{l}= \\
\text { 6. So. n. } \\
\text { Dreikőnig }\end{array}$ & $\begin{array}{l}= \\
\text { 5. So. }{ }^{7}\end{array}$ & $\begin{array}{l}= \\
\text { 5. So. }\end{array}$ \\
\hline
\end{tabular}

5 \{Katharina [25.11.]\}

- STEPHAN Beissel, Entstehung der Perikopen des Römischen MeBbuches, S. 172 u. S. 132, Anm. 5: 5. Sonntag nach Dreikönig.

7 Matthiss [24.2.]\} 


\section{XXVIII}

\begin{tabular}{|c|c|c|c|c|c|c|}
\hline Nr. & Tag in Hs. & Normtag & Fr & $\mathbf{B r}$ & N1 & $\mathbf{N 2}$ \\
\hline 36 & $\begin{array}{l}\text { Mittwoch n. } 5 . \\
\text { So. n. Dreikönig }\end{array}$ & $\begin{array}{l}\text { Fr. n. 4. So. n. } \\
\text { Dreikönig (N) }\end{array}$ & Mk 10, 13-16 & $\begin{array}{l}= \\
\text { Mittwoch }\end{array}$ & $\begin{array}{l}= \\
\text { Mittwoch }\end{array}$ & $\begin{array}{l}= \\
\text { Mittwoch }\end{array}$ \\
\hline 37 & $\begin{array}{l}\text { Freitag n. } 5 . \text { So. } \\
\text { n. Dreikönig }\end{array}$ & $\begin{array}{l}\text { Do. n. 2. Fasten- } \\
\text { sonntag (A) }\end{array}$ & Jo 6, 27-35 & $\begin{array}{l}= \\
\text { Freitag }\end{array}$ & $\begin{array}{l}= \\
\text { Freitag }\end{array}$ & $\begin{array}{l}= \\
\text { Freitag }\end{array}$ \\
\hline 38 & $\begin{array}{l}\text { Maria Lichtmeß } \\
\text { [2.2.] }\end{array}$ & & 0 & 0 & $\begin{array}{l}= \\
\text { (in Hs noch- } \\
\text { mals nach } \\
190 \text { ) }\end{array}$ & $\begin{array}{l}\text { Lk 2, 22-32 } \\
\text { Marienfesttag }\end{array}$ \\
\hline 39 & Septuagesima & & Mt 20, 1-14 & $=$ & $=$ & $=$ \\
\hline 40 & $\begin{array}{l}\text { Mittwoch n. } \\
\text { Septuagesima }\end{array}$ & - & Mk 5, 21-34 & $\begin{array}{l}= \\
\text { Mittwoch } \\
\text { (30-34 fehlt) }\end{array}$ & $\begin{array}{l}= \\
\text { Mittwoch } \\
\text { (30-34 fehlt) }\end{array}$ & $\begin{array}{l}= \\
\text { Mittwoch }\end{array}$ \\
\hline
\end{tabular}

41 Sexagesima Lk 8, 4-15 = =

So. n. Maria

Lichtmeß

\begin{tabular}{|c|c|c|c|c|c|}
\hline 42 & $\begin{array}{l}\text { Mittwoch nach } \\
\text { Sexagesima }\end{array}$ & Mt 12, 30-37 & $\begin{array}{l}= \\
\text { Mittwoch }\end{array}$ & $\begin{array}{l}= \\
\text { Mittwoch }\end{array}$ & $\begin{array}{l}= \\
\text { Mittwoch }\end{array}$ \\
\hline 43 & Quinquagesima & Lk 18, 31-43 & $=$ & $=$ & $=$ \\
\hline 44 & Aschermittwoch & Mt 6, 16-21 & $=$ & $=$ & $=$ \\
\hline 45 & $\begin{array}{l}\text { Do. } n \text {. Aschermitt- } \\
\text { woch (N) }\end{array}$ & $\circ$ & $\circ$ & $\begin{array}{l}\text { Mt 8, 5-13 } \\
\text { (vgl. 29: Mt 8, } \\
\text { 1-13) }\end{array}$ & $\circ$ \\
\hline 46 & $\begin{array}{l}\text { Freitag n. Ascher- } \\
\text { mittwoch }\end{array}$ & Mt $5,43-6,4$ & $\begin{array}{l}= \\
\text { Freitag }\end{array}$ & $\begin{array}{l}= \\
\text { Freitag }\end{array}$ & $\begin{array}{l}= \\
\text { Freitag }\end{array}$ \\
\hline 47 & Samstag & Mk 6, 47-56 & $=$ & $=$ & $=$ \\
\hline 48 & 1. Fastensonntag & Mt 4, 1-11 & $=$ & $=$ & $=$ \\
\hline 49 & Montag & $\begin{array}{l}\text { Mt } 25,31-46 \\
\text { (42-45 fehlt) }\end{array}$ & $\begin{array}{l}= \\
(42-45 \text { fehlt })\end{array}$ & $\begin{array}{l}= \\
(42-45 \text { fehlt })\end{array}$ & $\begin{array}{l}= \\
(42-45 \text { fehlt })\end{array}$ \\
\hline
\end{tabular}

- \{Freitag n. 5. So. n. Dreifaltigkeit\} 


\begin{tabular}{|c|c|c|c|c|c|c|}
\hline Nr. & Tag in Hs. & Normtag & $\mathbf{F r}$ & $\mathbf{B r}$ & N1 & $\mathbf{N 2}$ \\
\hline 50 & Dienstag & & Mt 21, 10-17 & $=$ & $=$ & $=$ \\
\hline 51 & Mittwoch & & Mt 12, 38-50 & $=$ & $=$ & $=$ \\
\hline 52 & Donnerstag & $(\mathrm{N})$ & Jo 8, 31-47 & $=$ & $=$ & $=$ \\
\hline 53 & Freitag & & Jo 5, 1-15 & $=$ & $=$ & $=$ \\
\hline 54 & Samstag & & Mt 17, 1-9 & $=$ & $=$ & $=$ \\
\hline 55 & 2. Fastensonntag & & Mt $15,21-28$ & $=$ & $=$ & $\begin{array}{l}= \\
\text { 2. So. }\end{array}$ \\
\hline 56 & $\begin{array}{l}\text { Montag n. } 2 . \\
\text { Fastensonntag }\end{array}$ & & Jo 8, 21-29 & $\begin{array}{l}= \\
\text { Montag }\end{array}$ & $\begin{array}{l}= \\
\text { Montag }\end{array}$ & $\begin{array}{l}= \\
\text { Montag }\end{array}$ \\
\hline 57 & Dienstag & & Mt 23, 1-12 & $=$ & $=$ & $=$ \\
\hline 58 & Mittwoch & & Mt 20, 17-28 & $=$ & $=$ & $=$ \\
\hline 59 & Donnerstag & (A) & $\begin{array}{l}\text { Lk 16, 19-31 } \\
\text { (vgl. Verweis } \\
128 \text { ) }\end{array}$ & $=$ & $=9$ & $\begin{array}{l}= \\
\text { (vgl. Verweis } \\
128)\end{array}$ \\
\hline 60 & Freitag & & $\begin{array}{l}\text { Mt 21, 33-46 } \\
\text { (39-46 fehlt) }\end{array}$ & $=$ & $=$ & $\begin{array}{l}= \\
\text { Sonntag }\end{array}$ \\
\hline 61 & - & $\begin{array}{l}\text { Sa. n. 2. Fasten- } \\
\text { sonntag }\end{array}$ & $\begin{array}{l}\text { Lk 15, 11-32 } \\
\circ \\
\text { (11-24 fehlt) }\end{array}$ & $\begin{array}{l}= \\
\text { Samstag }\end{array}$ & $\begin{array}{l}= \\
\text { Samstag }\end{array}$ & $\begin{array}{l}= \\
\text { Samstag }\end{array}$ \\
\hline 62 & 3. Fastensonntag & & Lk 11, 14-28 & $=$ & $=$ & $=$ \\
\hline 63 & Montag & & Lk 4, 23-30 & $=$ & $=$ & $=$ \\
\hline 64 & Dienstag & & Mt 18, 15-22 & $=$ & $=10$ & $=$ \\
\hline 65 & Mittwoch & & Mt 15, 1-20 & $\begin{array}{l}= \\
\text { (in Hs noch- } \\
\text { mals als Nach- } \\
\text { trag nach 223) }\end{array}$ & $=$ & $=$ \\
\hline
\end{tabular}

9 $\{1$. So. n. Dreifaltigkeit. Vgl. 128.

10 Petrus (Stuhlfeier) [22.2.]\} 


\begin{tabular}{|c|c|c|c|c|c|}
\hline Nr. & Tag in Hs. & $\mathbf{F r}$ & $\mathbf{B r}$ & N1 & $\mathbf{N 2}$ \\
\hline 66 & Donnerstag & $\begin{array}{l}\text { Lk 4, 38-43 } \\
\text { (vgl. 126) }\end{array}$ & $\begin{array}{l}= \\
\text { (vgl. 126) }\end{array}$ & $\begin{array}{l}= \\
\text { (vgl. 126) }\end{array}$ & $\begin{array}{l}= \\
\text { (vgl. 126) }\end{array}$ \\
\hline 67 & Freitag & Jo 4, 5-42 & $=$ & $=$ & $=$ \\
\hline 68 & Samstag & Jo 8, 1-11 & $=$ & $=$ & $=$ \\
\hline 69 & 4. Fastensonntag & Jo 6, 1-14 & $=$ & $=$ & $=$ \\
\hline 70 & Montag & Jo 2, 13-25 & $=$ & $=$ & $=$ \\
\hline 71 & Dienstag & Jo 7, 14-31 & $=$ & $=$ & $=$ \\
\hline 72 & Mittwoch & Jo 9, 1-38 & $\begin{array}{l}= \\
(17-25 \text { fehlt })\end{array}$ & $=$ & $=$ \\
\hline
\end{tabular}

73 Donnerstag

(A)

Lk 7, 11-16=

(vgl. 165) (vgl. 165) (vgl. 165) (vgl. Verweis 165)

\begin{tabular}{llllll}
\hline 74 & Freitag & Jo 11, 1-45 & $=$ & $=$ \\
\hline 75 & Samstag & Jo 8, 12-20 $=$ & $=$ & $=$ \\
\hline
\end{tabular}

$\begin{array}{llll}76 & 5 . \text { Fastensonntag } & \text { Jo 8, 46-59 } & =\end{array}$

\begin{tabular}{llllll}
77 & Montag & Jo 7, 32-39 & $=$ & $=$ \\
\hline 78 & Dienstag & Jo 7, 1-13 & $=$ & $=$ & $=$ \\
\hline 79 & Mittwoch & Jo 10, 22-38 & $=$ & $=$ & $=$ \\
\hline 80 & Donnerstag & (N) & Jo 7, 40-53 & $=$ & $=11$
\end{tabular}

\begin{tabular}{lllll} 
& & & Dienstag \\
\hline $81 \quad$ Freitag & Jo 11, 47-54 $=$ & $=$ \\
\hline
\end{tabular}

$\begin{array}{ll}\text { Samstag } & \begin{array}{l}\text { Samstag vor Palm- Jo 17, 1-26 } \\ \text { sonntag }(N)\end{array}\end{array}$

Samstag vor Samstag vor

Palmsonntag Palmsonntag

(13-26 fehlt) (13-26 fehlt)

1 \{Lk 7,36 als Anfang der hierhergehörenden Perikope - [P. PIETSCH (wie Anm. 1), S. 66: (A)] - mit Verweis auf Maria Magdalena [22.7.]\} Vgl. 189.

12 \{Vortag von Christi Himmelfahrt\} 


\begin{tabular}{|c|c|c|c|c|c|c|}
\hline Nr. & Tag in Hs. & Normtag & $\mathbf{F r}$ & $\mathbf{B r}$ & N1 & $\mathbf{N 2}$ \\
\hline 83 & Palmsonntag & (N) & $\begin{array}{l}\text { Lk 19, 29-40 } \\
\text { (Einschub v. } \\
\text { Me 21,8) }\end{array}$ & $=$ & $=13$ & $\begin{array}{l}\text { x } \\
\text { Samstag vor } \\
\text { Palmsonntag }\end{array}$ \\
\hline 84 & $\begin{array}{l}\text { Palmsonntag: } \\
\text { Passion }\end{array}$ & $\begin{array}{l}\text { Mt } 26,1-27,66 \\
\text { (N) }\end{array}$ & $\circ$ & $\circ$ & 0 & $\begin{array}{l}\text { Mt } 26,1-27,44 \\
\text { Mk } 15,33-46\end{array}$ \\
\hline 85 & $\begin{array}{l}\text { Montag n. Palm- } \\
\text { sonntag }\end{array}$ & $\begin{array}{l}\text { Evangelienteil der } \\
\text { Passion des Palm- } \\
\text { sonntag }\end{array}$ & Mt 27, 62-66 & $\begin{array}{l}= \\
\text { Montag }\end{array}$ & $\begin{array}{l}={ }^{14} \\
\text { Montag }\end{array}$ & $\begin{array}{l}= \\
\text { Montag }\end{array}$ \\
\hline 86 & Dienstag: Passion & (A) & $\circ$ & $\circ$ & $0^{15}$ & Mk $14,1-15,41$ \\
\hline 87 & Mittwoch: Passion & $n(\mathrm{~A})$ & 0 & $\circ$ & $0^{15}$ & Lk 22,1 - 23,53 \\
\hline 88 & Gründonnerstag & (A) & Jo 13, 1-38 & $=$ & $=16$ & $=$ \\
\hline 89 & $\begin{array}{l}\text { Karfreitag: } \\
\text { Passion }\end{array}$ & - & $\begin{array}{l}\text { Passions- } \\
\text { harmonie }\end{array}$ & $\circ$ & $\circ$ & $\circ$ \\
\hline 90 & $\begin{array}{l}\text { Karfreitag: } \\
\text { Passion }\end{array}$ & (A) & j & $\begin{array}{l}\text { Jo } 18,1-19,42 \\
(18,38-19,5 \\
\text { fehlt })\end{array}$ & $\begin{array}{l}= \\
(18,38-19,5 \\
\text { fehlt })\end{array}$ & $=$ \\
\hline 91 & $\begin{array}{l}\text { Samstag vor } \\
\text { Ostern }\end{array}$ & $\begin{array}{l}\text { Samstag n. Ostern } \\
\text { (vgl. 100) }\end{array}$ & Jo 20, 1-9 & $=$ & $=$ & $\begin{array}{l}= \\
\text { Samstag }\end{array}$ \\
\hline (23) & & & & (Jo 6, 55-59) & & \\
\hline 92 & Ostersonntag & & Mk 16, 1-7 & $=$ & $=$ & $=$ \\
\hline 93 & Montag & & Lk 24, 13-35 & $=$ & $=$ & $=$ \\
\hline (23) & & & & (Jo 6, 53-54) & & \\
\hline (224) & & & & (Mt 19, 23-26) & & \\
\hline
\end{tabular}

13 \{2. Adventssonntag\} Wohl bezogen auf die Parallelstelle Mt 21,1-9, die zum hier fehlenden Adventssonntag gehört.

14 UVerweis auf Jo 12,1 - [(A): Jo 12, 1-9; (N): Jo 12, 1-36] - als Evangelium für den Montag nach Palmsonntag\} Vgl. Nachtrag 226.

is Vgl. Eintrag späterer Hand in N1 bei 88.

16 \{Verweis auf Mk 14,1 als Passion am Dienstag, auf $L k 22,1$ als Passion am Mittwoch nach Palmsonntag\} Vgl. 86 und 87. 


\begin{tabular}{|c|c|c|c|c|c|c|}
\hline Nr. & Tag in Hs. & Normtag & $\mathbf{F r}$ & Br & N1 & N2 \\
\hline 94 & $\begin{array}{l}\text { Dienstag } \mathbf{n} . \\
\text { Ostern }\end{array}$ & & Lk 24, 36-47 & $\begin{array}{l}= \\
\text { Dienstag }\end{array}$ & $\begin{array}{l}= \\
\text { Dienstag }\end{array}$ & $\begin{array}{l}= \\
\text { Dienstag }\end{array}$ \\
\hline (218) & & & $(2 k 11,33-35)$ & & & \\
\hline 95 & $\begin{array}{l}\text { Mittwoch n. } \\
\text { Ostern }\end{array}$ & & Jo 21, 1-14 & $\begin{array}{l}= \\
\text { Mittwoch }\end{array}$ & $\begin{array}{l}= \\
\text { Mittwoch }\end{array}$ & $\begin{array}{l}= \\
\text { Mittwoch }\end{array}$ \\
\hline 96 & Donnerstag & & Jo 20, 11-18 & $=$ & $=$ & $=$ \\
\hline 97 & Freitag & $\begin{array}{l}\text { Freitag n. 1. So. } \\
\text { n. Ostern } \\
\text { (vgl. 102) }\end{array}$ & $\begin{array}{l}\text { Mt 28, 8-15 } \\
\text { (vgl. Verweis } \\
102)\end{array}$ & $\begin{array}{l}= \\
\text { (vgl. Verweis } \\
\text { 102) }\end{array}$ & $\begin{array}{l}=17 \\
\text { (vgl. Verweis } \\
102)\end{array}$ & $\begin{array}{l}= \\
\text { (in Hs noch- } \\
\text { mals bei 102) }\end{array}$ \\
\hline 98 & Samstag & & Mt 28, 16-20 & $=$ & $\begin{array}{l}=18 \\
\text { Freitag getilgt }\end{array}$ & $\begin{array}{l}= \\
\text { Mittwoch }\end{array}$ \\
\hline 99 & 1. So. n. Ostern & & Jo $20,19-31$ & $=$ & $=19$ & $=$ \\
\hline 100 & $\begin{array}{l}\text { Montag n. 1. So. } \\
\text { n. Ostern }\end{array}$ & $\begin{array}{l}\text { Samstag vor } \\
\text { Ostern } \\
\text { (vgl. 91) }\end{array}$ & Mt 28, 1-7 & $\begin{array}{l}= \\
\text { Samstag vor } \\
\text { Ostern }\end{array}$ & $\begin{array}{l}={ }^{20} \\
\text { Samstag n. } \\
\text { Ostern }\end{array}$ & $\begin{array}{l}= \\
\text { Samstag vor } \\
\text { Ostern }\end{array}$ \\
\hline 101 & Mittwoch & & Mk 16, 9-13 & $=$ & $=$ & $=$ \\
\hline 102 & $\begin{array}{l}\text { Freitag n. } 2 . \text { So. } \\
\text { n. Ostern }\end{array}$ & $\begin{array}{l}\text { Freitag n. 1. So. } \\
\text { n. Ostern } \\
\text { (vgl. 97) }\end{array}$ & $\begin{array}{l}\text { Mt 28, } 8 \\
\text { (Verweis auf } \\
\text { 97) }\end{array}$ & $\begin{array}{l}= \\
\text { Freitag } \\
\text { (Verweis auf } \\
\text { 97) }\end{array}$ & $\begin{array}{l}=21 \\
\text { Freitag } \\
\text { (Verweis auf } \\
\text { 97) }\end{array}$ & $\begin{array}{l}\text { (Mt 28, 8-15) } \\
\text { Freitag } \\
\text { (gestellt zu 97) }\end{array}$ \\
\hline 103 & 2. So. n. Ostern & & Jo $10,11-16$ & $\begin{array}{l}= \\
\text { 2. Sonntag }\end{array}$ & $\begin{array}{l}= \\
\text { 2. Sonntag }\end{array}$ & $\begin{array}{l}= \\
\text { 2. Sonntag }\end{array}$ \\
\hline 104 & Mittwoch & & Lk 24, 1-12 & $=$ & $=$ & $=$ \\
\hline 105 & Freitag & $\begin{array}{l}\text { Freitag n. 3. So. } \\
\text { n. Ostern }\end{array}$ & Jo $12,44-50$ & $=$ & $=n$ & $=$ \\
\hline \multicolumn{7}{|c|}{$\begin{array}{ll}17 & \text { \{Freitag n. 1. Sonntag } \\
18 & \text { \{Freitag\} } \\
19 & \text { \{Samstag, aber wieder } \\
\text { 20 } & \text { \{Freitag, aber wieder 8 } \\
21 & \text { \{Freitag n. Ostern } \\
2 & \{\text { Mt 9,14 gehöre hierhe } \\
\end{array}$} \\
\hline
\end{tabular}




\begin{tabular}{|c|c|c|c|c|c|c|}
\hline Nr. & Tag in Hs. & Normtag & Fr & $\mathbf{B r}$ & N1 & N2 \\
\hline 106 & 3. So. n. Ostern & & Jo $16,16-22$ & $=$ & $=$ & $=$ \\
\hline 107 & Mittwoch & & Jo 3, 25-36 & $=$ & $=$ & $=$ \\
\hline 108 & 4. So. n. Ostern & & Jo 16, 5-15 & $\begin{array}{l}= \\
\text { 4. Sonntag }\end{array}$ & $\begin{array}{l}=\sqrt{3} \\
\text { 4. Sonntag }\end{array}$ & $=$ \\
\hline 109 & Mittwoch & $\begin{array}{l}\text { Freitag n. 4. So. } \\
\text { n. Ostern }\end{array}$ & Jo $13,33-36$ & $=$ & $=$ & $\begin{array}{l}= \\
\text { Freitag }\end{array}$ \\
\hline 110 & Freitag & $\begin{array}{l}\text { Vortag v. Philip- } \\
\text { pus und Jakobus } \\
\text { [30.4.] } \\
\text { (vgl. auch 118) }\end{array}$ & Jo 15, 1-11 & $=$ & $=24$ & $\begin{array}{l}= \\
\text { Tiburtius und } \\
\text { Valerianus } \\
\text { [14.4.] } \\
\text { (vgl. 118) }\end{array}$ \\
\hline 111 & Philippus [1.5.] & & Jo $14,1-14$ & $\begin{array}{l}= \\
\text { 5. Sonntag }\end{array}$ & $\begin{array}{l}=25 \\
5 \text {. Sonntag } \\
(10-14 \text { fehlt })\end{array}$ & $\begin{array}{l}= \\
\text { Philippus und } \\
\text { Jakobus [1.5.] }\end{array}$ \\
\hline 112 & $\begin{array}{l}\text { Kreuzaufrindung } \\
\text { [3.5] }\end{array}$ & & $\begin{array}{l}\text { Jo 3, } 1 \\
\text { (Verweis auf } \\
\text { 127) }\end{array}$ & $\begin{array}{l}= \\
\text { Nerweis auf } \\
\text { 127) }\end{array}$ & $\begin{array}{l}\circ \\
\text { (vgl. 127) }\end{array}$ & $\begin{array}{l}(=) \\
\text { (in Hs hier Jo 3, } \\
\text { 1-15; Verweis in } \\
\text { 127) }\end{array}$ \\
\hline
\end{tabular}

113 5. So. n. Ostern

Jo $16,23-30=$

- $=$

\section{Sonntag}

5. Sonntag

\begin{tabular}{|c|c|c|c|c|c|c|}
\hline 114 & Mittwoch & $\begin{array}{l}\text { Montag n. 5. So. } \\
\text { n. Ostern }\end{array}$ & Lk 11, 5-13 & $=$ & $0^{20}$ & $\begin{array}{l}= \\
\text { Markus [25.4.] }\end{array}$ \\
\hline 115 & $\begin{array}{l}\text { Christi Himmel- } \\
\text { fahrt }\end{array}$ & & Mk 16, 14-20 & $\begin{array}{l}= \\
\text { Himmelfahrt }\end{array}$ & $={ }^{27}$ & $\begin{array}{l}= \\
\text { Himmelfahrt }\end{array}$ \\
\hline
\end{tabular}

23 \{Jo 12,44 mit Verweis auf 105, das hierhergehöre\}

24 \{Oder Tiburtius und Valerianus [14.4.] sowie Vortag von Philippus und Jakobus [30.4.]; Festtag Philippus und Jakobus [1.5.] getilgt\}

25 Philippus und Jakobus [1.5.]\}

20 \{Montag vor Christi Himmelfahrt - [vgl. H. GroTEFEND (wie Anm. 2) Bd. I, S. 108] - mit Verweis: stett do hinten\} Fehlt aber in der Handschrift.

27 \{Auch Aussendung der Apostel [15.7.] - [tayddung wohl Verschreibung fur taylung. Vgl. H. GroTEFEND (wie Anm. 2) Bd. I, S. 12] -; Verweis auf Jo 17,1 als Evangelium vom Vortag von Christi Himmelfahrt\} Vgl. 117. 


\section{XXXIV}

\begin{tabular}{|c|c|c|c|c|c|c|}
\hline Nr. & Tag in Hs. & Normtag & $\mathbf{F r}$ & $\mathbf{B r}$ & N1 & $\mathbf{N 2}$ \\
\hline 116 & $\begin{array}{l}\text { Sonntag n. Chri- } \\
\text { sti Fimmelfahrt }\end{array}$ & & Jo $15,26-16,4$ & $\begin{array}{l}= \\
\text { Sonntag nach } \\
\text { Himmelfahrt }\end{array}$ & $=x$ & $\begin{array}{l}= \\
\text { Sonntag nach } \\
\text { Himmelfahrt }\end{array}$ \\
\hline 117 & Mittwoch & (N) & Lk 24, 49-53 & $=$ & $=$ & $=$ \\
\hline 118 & Freitag & & $\circ$ & $\circ$ & $\circ$ & $\begin{array}{l}\text { Jo 15, 7-11 } \\
\text { (vgl. 110) }\end{array}$ \\
\hline 119 & $\begin{array}{l}\text { Samstag vor } \\
\text { Pfingsten }\end{array}$ & & Jo $14,15-21$ & $=$ & $=$ & $=$ \\
\hline 120 & Pringsten & & Jo $14,23-31$ & $=$ & $=$ & $=$ \\
\hline 121 & $\begin{array}{l}\text { Montag n. } \\
\text { Pfingsten }\end{array}$ & & Jo $3,16-21$ & $\begin{array}{l}= \\
\text { Montag }\end{array}$ & $\begin{array}{l}= \\
\text { Montag }\end{array}$ & $\begin{array}{l}= \\
\text { Montag }\end{array}$ \\
\hline 122 & Dienstag & & Jo $10,2-10$ & $=$ & $=$ & $=$ \\
\hline 123 & Mittwoch & & Jo 6, 44-52 & $=$ & $=20$ & $=$ \\
\hline 124 & Donnerstag & $\begin{array}{l}\text { Freitag n. } \\
\text { Pfingsten }\end{array}$ & Lk 5, 17-26 & $=$ & $\begin{array}{l}(=) \\
\text { (in Hs hier 125: } \\
L \times 9,1-6)\end{array}$ & $\begin{array}{l}(=) \\
\text { (in Hs hier 125: } \\
L \times 9,1-6)\end{array}$ \\
\hline 125 & Freitag & $\begin{array}{l}\text { Donnerstag n. } \\
\text { Pfingsten }\end{array}$ & Lk 9, 1-6 & $=$ & $\begin{array}{l}(=) \\
\text { (in Hs hier 124: } \\
\text { Lk } 5,17-26)\end{array}$ & $\begin{array}{l}(=) \\
\text { (in Hs hier 125: } \\
L k 9,17-26)\end{array}$ \\
\hline 126 & Samstag & (N) & $\begin{array}{l}\text { Lk 4, 38-43 } \\
\text { (vgl. 66) }\end{array}$ & $\begin{array}{l}= \\
\text { (vgl 66) }\end{array}$ & $\begin{array}{l}= \\
\text { (vgl. 66) }\end{array}$ & $\begin{array}{l}= \\
\text { (vgl. 66) }\end{array}$ \\
\hline 127 & $\begin{array}{l}\text { 1. So. n. } \\
\text { Pringsten / Drei- } \\
\text { faltigkeit }\end{array}$ & (A) & Jo 3, 1-15 & $=$ & $=\mathbf{3}^{\mathbf{3 0}}$ & $\begin{array}{l}(=) \\
\text { (Jo 3,1 mit } \\
\text { Verweis auf } \\
\text { 112: Jo 3,1-15) }\end{array}$ \\
\hline 128 & $\begin{array}{l}\text { 1. So. n. } \\
\text { Dreifaltigkeit }\end{array}$ & & $\begin{array}{c}\text { Lk 16, } 19 \\
\text { (Verweis auf 59) }\end{array}$ & $\circ$ & $\begin{array}{c}\circ \\
\text { (vgl. } 59 \text { u. 131) }\end{array}$ & $\begin{array}{l}= \\
\circ \\
\text { (Verweis auf 59) }\end{array}$ \\
\hline
\end{tabular}

2 (Verweis auf Lk 24,49 als Evangelium für Freitag nach Christi Himmelfahrt\} Vgl. 117.

- (Quatember in der Pfingstwoche\}

*o \{Kreuzauffindung\} Vgl. 112. 


\begin{tabular}{|c|c|c|c|c|c|c|}
\hline Nr. & Tag in Hs. & Normtag & $\mathbf{F r}$ & $\mathbf{B r}$ & N1 & N2 \\
\hline 129 & Mittwoch & $\begin{array}{l}\text { Freitag } n . \text { Dreifal- } \\
\text { tigkeit (A) }\end{array}$ & & $\circ$ & $\begin{array}{l}\circ \\
\text { (vgl 132) }\end{array}$ & Lk 20, 27-40 \\
\hline 130 & $\begin{array}{l}\text { Freitag n. 1. So. } \\
\text { n. Dreifaltigkeit }\end{array}$ & $\begin{array}{l}\text { Fr. n. 2. So. n. } \\
\text { Dreifaltigkeit }\end{array}$ & Lk 8, $41-56$ & $\begin{array}{l}= \\
\text { Freitag }\end{array}$ & $\begin{array}{l}\circ \\
\text { (vgl. 132) }\end{array}$ & $\begin{array}{l}= \\
\text { Freitag }\end{array}$ \\
\hline 131 & $\begin{array}{l}\text { 2. So. n. } \\
\text { Pfingsten }\end{array}$ & $\begin{array}{l}\text { 2. So. n. } \\
\text { Dreifaltigkeit }\end{array}$ & Lk $14,16-24$ & $\begin{array}{l}= \\
\text { 2. Sonntag }\end{array}$ & $\begin{array}{l}(=) \\
\text { (in Hs hier 132: } \\
\text { Lk 15, 1-10) }\end{array}$ & $=$ \\
\hline 132 & $\begin{array}{l}\text { 3. So. n. } \\
\text { Pfingsten }\end{array}$ & $\begin{array}{l}\text { 3. So. n. } \\
\text { Dreifaltigkeit }\end{array}$ & Lk 15, 1-10 & $\begin{array}{l}= \\
\text { 3. Sonntag }\end{array}$ & $\begin{array}{l}(\Rightarrow) \\
\text { (in Hs hier 131: } \\
\text { Lk 14,16-24) }\end{array}$ & $=$ \\
\hline 133 & Mittwoch & $\begin{array}{l}\text { Mi. n. 3. So. n. } \\
\text { Dreifaltigkeit }\end{array}$ & Mt 5, 25-29 & $=$ & $=$ & $=$ \\
\hline 134 & Freitag & $\begin{array}{l}\text { Fr. n. 3. So. n. } \\
\text { Dreifaltigkeit }\end{array}$ & Mk 11, 11-18 & $=$ & $={ }^{3}$ & $=$ \\
\hline 135 & $\begin{array}{l}\text { 4. So. n. } \\
\text { Pfingsten }\end{array}$ & $\begin{array}{l}\text { 4. So. } \mathrm{n} \text {. } \\
\text { Dreifaltigkeit }\end{array}$ & Lk 6, 36-42 & $\begin{array}{l}= \\
\text { 4. Sonntag }\end{array}$ & $={ }^{34}$ & $\begin{array}{l}= \\
\text { 4. Sonntag }\end{array}$ \\
\hline 136 & Mittwoch & $\begin{array}{l}\text { Mi. n. 4. So. n. } \\
\text { Dreifaltigkeit }{ }^{35}\end{array}$ & $\begin{array}{l}\text { Mt 21, 23-32; } \\
11,15\end{array}$ & $=$ & $\circ$ & $\begin{array}{l}= \\
0\end{array}$ \\
\hline 137 & Freitag & $\begin{array}{l}\text { Fr. n. 4. So. n. } \\
\text { Dreifaltigkeit (A) }\end{array}$ & Mt $17,10-18$ & $=$ & $=$ & $=$ \\
\hline 138 & $\begin{array}{l}\text { 5. So. n. } \\
\text { Pfingsten }\end{array}$ & $\begin{array}{l}\text { 5. So. } \mathbf{n} \text {. } \\
\text { Dreifaltigkeit }\end{array}$ & Lk 5, 1-11 & $\begin{array}{l}= \\
\text { 5. Sonntag }\end{array}$ & $\begin{array}{l}= \\
\text { 5. Sonntag }\end{array}$ & $\begin{array}{l}= \\
\text { 5. Sonntag }\end{array}$ \\
\hline
\end{tabular}

31 Dazu \{3. Sonntag n. Dreifaltigkeit; außerdem: $\mathbf{k}$ 15,1 mit Verweis auf Mittwoch n. Dreifaltigkeit [vgl. 132]; Jo 6,56 mit Verweis auf Vortag und Fest Fronleichnam [vgl. 23, wo es in N1 aber fehlt.]; Verweis auf 1. Sonntag n. Dreifaltigkeit [vgl. 59]\}

32 Daxu \{2. Sonntag n. Dreifaltigkeit; außerdem: Mt 11,20 mit Verweis auf Mittwoch n. 1. Sonntag n. Dreifaltigkeit [vgl. 129, steht aber in 158]; $L k 14,1$ (oder Lk 6,6) mit Verweis auf Freitag $n$. 1. Sonntag $n$. Dreifaltigkeit [vgl. 130, steht aber in 167]\}

33 Mittwoch n. 14. Sonntag nach Ostern (!)\}

4 $\{$ k 9,12 mit Verweis auf Mittwoch n. 3. Sonntag n. Dreifaltigkeit [vgl. 180]; Lk 8,41 mit Verweis auf Freitag n. 3. Sonntag n. Dreifaltigkeit [vgl. 130]\}

3s Vgl. P. PIETSCH (wie Anm. 1), S. 70: A/N: Mt 21, 23-27; Sp: Mt 21, 23-32. 


\begin{tabular}{lllllll} 
Nr. & Tag in Hs. & Normtag & Fr & Br & N1 & N2 \\
\hline 139 & 6. Sonntag & $\begin{array}{l}\text { 6. So. n. } \\
\text { Dreifaltigkeit }\end{array}$ & Mt 5, 20-24 $=$ & $=$ \\
\hline
\end{tabular}

140 Mittwoch Mi.n. 6. So. n. Mk 10, 17-21 $=$ =

Dreifaltigkeit

\begin{tabular}{|c|c|c|c|c|c|c|}
\hline 141 & Freitag & -36 & Mk 8, 11-26 & 0 & 0 & $=$ \\
\hline 142 & $\begin{array}{l}\text { 7. So. n. } \\
\text { Pringsten }\end{array}$ & $\begin{array}{l}\text { 7. So. } n \text {. } \\
\text { Dreifaltigkeit }\end{array}$ & Mk 8, 1-9 & $\begin{array}{l}=37 \\
7 . \text { Sonntag }\end{array}$ & $\begin{array}{l}= \\
\text { 7. Sonntag }\end{array}$ & $\begin{array}{l}= \\
\text { 7. Sonntag }\end{array}$ \\
\hline
\end{tabular}

143 Mittwoch Mi.n. 7. So. n. Mt 16, 1-6 $=$ =

Dreifaltigkeit ${ }^{38}$

\begin{tabular}{|c|c|c|c|c|c|c|}
\hline 144 & Freitag & -30 & Lk 9, 37-43 & $=$ & $=$ & $=$ \\
\hline 145 & $\begin{array}{l}\text { 8. So. n. } \\
\text { Pringsten }\end{array}$ & $\begin{array}{l}\text { 8. So. } n \text {. } \\
\text { Dreifaltigkeit }\end{array}$ & Mt 7, 15-21 & $\begin{array}{l}= \\
\text { 8. Sonntag }\end{array}$ & $\begin{array}{l}= \\
\text { 8. Sonntag }\end{array}$ & $\begin{array}{l}= \\
\text { 8. Sonntag }\end{array}$ \\
\hline 146 & Mittwoch & $-\infty$ & Lk 8, 27-39 & $=$ & $\circ$ & $=$ \\
\hline 147 & Freitag & $\begin{array}{l}\text { Fr. n. 8. So. n. } \\
\text { Dreifaltigkeit }\end{array}$ & Mt 23, 13-23 & $\circ$ & o & $=$ \\
\hline 148 & $\begin{array}{l}\text { 9. So. n. } \\
\text { Pfingsten }\end{array}$ & $\begin{array}{l}\text { 9. So. n. } \\
\text { Dreifaltigkeit }\end{array}$ & Lk 16, 1-9 & $\begin{array}{l}= \\
0\end{array}$ & $=$ & $\begin{array}{l}= \\
\text { 9. Sonntag }\end{array}$ \\
\hline 149 & Mittwoch & $\begin{array}{l}\text { Mi n. 9. So. n. } \\
\text { Dreifaltigkeit (A) }\end{array}$ & Lk 21, 20-26 & $=$ & $=$ & $=$ \\
\hline 150 & Freitag & $\begin{array}{l}\text { Fr. n. 9. So. n. } \\
\text { Dreifaltigkeit (A) }\end{array}$ & Lk 21, 34-36 & $=$ & $=$ & $=$ \\
\hline 151 & $\begin{array}{l}\text { 10. So. } n . \\
\text { Pfingsten }\end{array}$ & $\begin{array}{l}\text { 10. So. } n \text {. } \\
\text { Dreifaltigkeit }\end{array}$ & Lk 19, $41-47$ & $\begin{array}{l}= \\
\text { 10. Sonntag }\end{array}$ & $=$ & $\begin{array}{l}= \\
\text { 10. Sonntag }\end{array}$ \\
\hline
\end{tabular}

6gl. P. PIETsCh (wie Anm. 1), S. 70: Freitag n. 6. Sonntag n. Dreifaltigkeit: Mk 5, 1-20 (A/N); Mk 8, 11-26 (Sp).

37 Nachtrag 6. Hand (Perikopenverzeichnis) stellt dies Evangelium (Mk 8, 1-9) zum 5. Sonntag n. Pfingsten.

* Vgl. P. PIETSCH (wie Anm. 1), S. 70: A/N: Mt 16, 1-12; Sp: Mt 16, 1-6.

39 Vgl. P. PIETSCH (wie Anm. 1), S. 70: Freitag n. 7. Sonntag n. Dreifaltigkeit: Lk 9, 37-43 (Sp).

- Vgl. P. PIETsCh (wie Anm. 1), S. 70: Mittwoch n. 8. Sonntag n. Dreifaltigkeit: Lk 8, 27-35 (Sp). 


\begin{tabular}{|c|c|c|c|c|c|c|}
\hline Nr. & Tag in Fs. & Normtag & $\mathbf{F r}$ & $\mathbf{B r}$ & N1 & N2 \\
\hline 152 & Mittwoch & $\begin{array}{l}\text { Mi. n. 10. So. n. } \\
\text { Dreifaltigkeit (A) }\end{array}$ & Mt $14,15-21$ & $=$ & $=$ & $=$ \\
\hline 153 & $\begin{array}{l}\text { 11. So. n. } \\
\text { Pringsten }\end{array}$ & $\begin{array}{l}\text { 11. So. n. } \\
\text { Dreifaltigkeit }\end{array}$ & Lk 18, 9-14 & $\begin{array}{l}= \\
\text { 11. Sonntag }\end{array}$ & $=$ & $\begin{array}{l}= \\
\text { 11. Sonntag }\end{array}$ \\
\hline 154 & $\begin{array}{l}\text { 12. So. n. } \\
\text { Pfingsten }\end{array}$ & $\begin{array}{l}\text { 12. So. n. } \\
\text { Dreifaltigkeit }\end{array}$ & Mk 7, 31-37 & $\begin{array}{l}= \\
\text { 12. Sonntag }\end{array}$ & $\begin{array}{l}= \\
\text { 12. Sonntag }\end{array}$ & $\begin{array}{l}= \\
\text { 12. Sonntag }\end{array}$ \\
\hline 155 & $\begin{array}{l}\text { Maria Magdalena } \\
\text { Vor- und Festtag } \\
\text { [21./ 22.7.] }\end{array}$ & & $\begin{array}{l}\text { Lk 7, } 36 \\
\text { (Verweis auf } \\
189)\end{array}$ & 0 & ० & $\circ$ \\
\hline 156 & $\begin{array}{l}\text { 13. So. n. } \\
\text { Pringsten }\end{array}$ & $\begin{array}{l}\text { 13. So. n. } \\
\text { Dreifaltigkeit }\end{array}$ & Lk 10, 23-37 & $\begin{array}{l}= \\
\text { 13. Sonntag }\end{array}$ & $=$ & $\begin{array}{l}= \\
\text { 13. Sonntag }\end{array}$ \\
\hline 157 & Mittwoch & $\begin{array}{l}\text { Mi. n. 13. So. n. } \\
\text { Dreifaltigkeit (A) }\end{array}$ & Lk 13, 22-29 & $=$ & $=$ & $=$ \\
\hline 158 & Freitag & $\begin{array}{l}\text { Fr. n. 11. So n. } \\
\text { Dreifaltigkeit (A) }\end{array}$ & Mt 11, 20-24 & $=$ & $=$ & $=$ \\
\hline 159 & 14. Sonntag & $\begin{array}{l}\text { 14. So. n. } \\
\text { Dreifaltigkeit }\end{array}$ & Lk 17, 11-19 & $=$ & $\begin{array}{l}= \\
\text { 14. Sonntag } n . \\
\text { Pfingsten }\end{array}$ & $=$ \\
\hline 160 & Freitag & - & Lk 19, 11-28 & $=$ & o & $=$ \\
\hline 161 & 15. Sonntag & $\begin{array}{l}\text { 15. So. n. } \\
\text { Dreifaltigkeit }\end{array}$ & Mt 6, 24-33 & $=$ & $\begin{array}{l}= \\
\text { 15. Sonntag } n . \\
\text { Pfingsten }\end{array}$ & $=$ \\
\hline 162 & Mittwoch & $\begin{array}{l}\text { Mi n. 16. So. n. } \\
\text { Dreifaltigkeit (A) } \\
\text { [Quatember] }\end{array}$ & Mk 9, 17-29 & $\circ$ & o & $=$ \\
\hline 163 & Freitag & $\begin{array}{l}\text { Fr. n. 16. So. n. } \\
\text { Dreifaltigkeit (A) } \\
\text { [Quatember] }\end{array}$ & Lk 18, $1-8$ & $=$ & $\begin{array}{l}= \\
\text { Mittwoch }\end{array}$ & $=$ \\
\hline 164 & $\begin{array}{l}\text { Quatembersams- } \\
\text { tag }\end{array}$ & $\begin{array}{l}\text { Sa. n. 16. So. n. } \\
\text { Dreifaltigkeit (A) } \\
\text { [Quatember] }\end{array}$ & Lk 13, 6-17 & $=$ & $=$ & $\begin{array}{l}= \\
\text { Samstag }\end{array}$ \\
\hline
\end{tabular}

4 Vgl. P. PIETSCH (wie Anm. 1), S. 70: (N) Freitag n. 12. Sonntag n. Dreifaltigkeit. 


\begin{tabular}{|c|c|c|c|c|c|c|}
\hline Nr. & Tag in Hs. & Normtag & $\mathbf{F r}$ & $\mathbf{B r}$ & N1 & N2 \\
\hline 165 & $\begin{array}{l}\text { 16. So. n. } \\
\text { Pringsten }\end{array}$ & $\begin{array}{l}\text { 16. So. } \mathbf{n} \text {. } \\
\text { Dreifaltigkeit }\end{array}$ & $\begin{array}{l}\text { Lk 7, 11-16 } \\
\text { (vgl. 73) }\end{array}$ & $\begin{array}{l}= \\
\text { 16. Sonntag } \\
\text { (vgl. 73) }\end{array}$ & $\begin{array}{l}= \\
\text { (vgl. 73) }\end{array}$ & $\begin{array}{l}\text { Lk 7, } 11 \\
\text { (Verweis auf 73) }\end{array}$ \\
\hline 166 & Mittwoch & $\begin{array}{l}\text { Mi. n. 2. Advents- } \\
\text { sonntag (N) }\end{array}$ & - Mt 11, 11-15 & $=$ & $=2$ & $=$ \\
\hline 167 & 17. Sonntag & $\begin{array}{l}\text { 17. So. n. } \\
\text { Dreifaltigkeit }\end{array}$ & Lk 14, 1-11 & $=$ & $\begin{array}{l}= \\
\text { 17. Sonntag } n . \\
\text { Pfingsten }\end{array}$ & $=$ \\
\hline 168 & 18. Sonntag & $\begin{array}{l}\text { 18. So. n. } \\
\text { Dreifaltigkeit }\end{array}$ & Mt 22, 34-46 & $=$ & $\begin{array}{l}= \\
\text { 18. Sonntag } n . \\
\text { Pfingsten }\end{array}$ & $=$ \\
\hline 169 & Mittwoch & $\begin{array}{l}\text { Mi. n. 3. Advents- } \\
\text { sonntag }\end{array}$ & $\begin{array}{l}\text { - Lk 1, } 26 \\
\text { (Verweis auf 3) }\end{array}$ & $\begin{array}{l}= \\
\text { (Verweis auf 3) }\end{array}$ & $\begin{array}{l}={ }^{4} \\
\text { (Verweis auf 3) }\end{array}$ & $\begin{array}{l}\text { Ik 1, 26-38) } \\
\text { (gestellt zu 5) }\end{array}$ \\
\hline 170 & Freitag & $\begin{array}{l}\text { Fr. n. 3. Advents- } \\
\text { sonntag }\end{array}$ & $\begin{array}{l}\text { Lk 1, } 39 \\
\text { (Venweis auf 6) }\end{array}$ & $\begin{array}{l}= \\
\text { (Verweis auf 6) }\end{array}$ & $\begin{array}{l}= \\
\text { (Venweis auf 6) }\end{array}$ & $\begin{array}{l}(L k 1,39-47) \\
\text { (gestellt } z u 7)\end{array}$ \\
\hline 171 & 19. Sonntag & -45 & Mt 13, 24-30 & $=$ & $=$ & $=$ \\
\hline 172 & 20. Sonntag & & Mt 9, 1-8 & $\begin{array}{l}\text { (=) } \\
20 . \text { Sonntag n. } \\
\text { Pfingsten } \\
\text { (in Hs als Nach- } \\
\text { trag nach 222) }\end{array}$ & $=$ & $=$ \\
\hline 173 & & $\begin{array}{l}\text { Mi. n. 20. So. } n \text {. } \\
\text { Dreifaltigkeit }\end{array}$ & $\circ$ & $\begin{array}{l}\text { Lk 14, 12-15 } \\
\text { 20. Sonntag }\end{array}$ & $\begin{array}{l}= \\
\text { Mittwoch }\end{array}$ & $\begin{array}{l}= \\
\text { Mittwoch }\end{array}$ \\
\hline 174 & Freitag & $\begin{array}{l}\text { Fr. n. 20. So. } n \text {. } \\
\text { Dreifaltigkeit (A) }\end{array}$ & 0 & Mt 13, 31-35 & $=$ & $=$ \\
\hline 175 & 21. Sonntag & (A) & Mt 22, 1-14 & $=$ & $\begin{array}{l}= \\
\text { 21. Sonntag n. } \\
\text { Pfingsten }\end{array}$ & $=$ \\
\hline
\end{tabular}

2 Auch Mittwoch n. 2. Adventssonntag\}

- Auch Maria Verkūndigung [25.3.].

4 Maria Verkündigung [25.3.]\}

\& Vgl. P. PIETSCH (wie Anm. 1), S. 71: 19. Sonntag n. Dreifaltigkeit: Mt 13, 24-30 (Sp). 


\begin{tabular}{|c|c|c|c|c|c|c|}
\hline Nr. & Tag in Hs. & Normtag & $\mathbf{F r}$ & Br & N1 & N2 \\
\hline 176 & 22. Sonntag & & Jo 4, 46-53 & $=$ & $\begin{array}{l}= \\
\text { 22. Sonntag } n \text {. } \\
\text { Pfingsten }\end{array}$ & $=$ \\
\hline 177 & 23. So. & & Mt 18, 23-35 & $=$ & $\begin{array}{l}= \\
\text { 23. Sonntag } n \text {. } \\
\text { Pfingsten }\end{array}$ & $=$ \\
\hline 178 & 24. So. & & Mt 22, 15-21 & $=$ & $\begin{array}{l}= \\
\text { 24. Sonntag } n \text {. } \\
\text { Pfingsten }\end{array}$ & $=$ \\
\hline 179 & 25. So. & & Mt 9, 18-22 & $=$ & $\begin{array}{l}= \\
25 . \text { Sonntag } n . \\
\text { Pfingsten }\end{array}$ & $\begin{array}{l}= \\
\text { Mittwoch }\end{array}$ \\
\hline 180 & Mittwoch & $\begin{array}{l}\text { Mi. n. 2. So. n. } \\
\text { Dreifaltigkeit }{ }^{4}\end{array}$ & Lk 9, 12-17 & $=$ & $=$ & $\begin{array}{l}= \\
\text { Freitag }\end{array}$ \\
\hline
\end{tabular}

\section{SANKTORALE}

Proprium

\begin{tabular}{|c|c|c|c|c|c|}
\hline 181 & -47 & $\begin{array}{l}\text { Lk 12, 11-21 } \\
\text { Beginn des } \\
\text { Sanktorale }\end{array}$ & $\begin{array}{l}= \\
\text { Beginn des } \\
\text { Sanktorale }\end{array}$ & $\begin{array}{l}= \\
\text { Beginn des } \\
\text { Sanktorale }\end{array}$ & $\begin{array}{l}= \\
\text { Beginn des } \\
\text { Sanktorale }\end{array}$ \\
\hline 182 & & $\circ$ & $\begin{array}{l}\text { Lk 12, 13-21 } \\
0 \\
\text { (in Hs als Nach- } \\
\text { trag nach 225) }\end{array}$ & . & 0 \\
\hline 183 & $\begin{array}{l}\text { Johannes d. T. } \\
\text { Vortag [23.6.] }\end{array}$ & Lk 1, 5-17 & $=$ & $=$ & $\begin{array}{l}= \\
\text { Johannes Vortag }\end{array}$ \\
\hline 184 & $\begin{array}{l}\text { Johannes d. T. } \\
\text { [24.6.] }\end{array}$ & Lk 1, 57-68 & $=$ & $=$ & $\begin{array}{l}= \\
0\end{array}$ \\
\hline
\end{tabular}

4 Vgl. P. PIETSCH (wie Anm. 1), S. 69: Mittwoch n. Dreifaltigkeit: Lk 12, 11-21 (A); L 9,12-16 (Sp).

n Vgl. P. PIETSCH (wie Anm. 1), S. 69: Lk 12, 11-21: Mittwoch n. Dreifaltigkeit (A); Freitag nach Dreifaltigkeit (Sp). Vgl. 180. 
XL

\begin{tabular}{|c|c|c|c|c|c|}
\hline Nr. & Tag in Hs. & $\mathbf{F r}$ & $\mathbf{B r}$ & $\mathbf{N 1}$ & $\mathbf{N 2}$ \\
\hline 185 & $\begin{array}{l}\text { Petrus und Paulus } \\
\text { Vortag [28.6.] }\end{array}$ & Jo 21, 15-19 & $=$ & $=$ & $\begin{array}{l}\quad= \\
\text { Petrus und Paulus } \\
\text { Festtag }\end{array}$ \\
\hline
\end{tabular}

186 Petrus und Paulus

Mt 16, 13-19 = = = [29.6.]

187 Paulus (Gedilcht-

Mt 19, 27-29 0

nisfeier) [30.6.]

188 Petrus und Paulus -

Oktavtag [6.7.]

Mt 14, 22-33=

Petrus

189 Maria Magda- (N)

lena [22.7.]

Lk 7, 36-47 =

(vgl. Verweis 155)

190 Sieben Brüder

Mt 12, 46-50 。

(10.7.)

(38)

(Lk 2, 22-32)

191 Laurentius

Jo 12, 24-26

$={ }^{51}$

[10.8.]

192 Maria Himmel-

fahrt [15.8.]

Lk 10, 38-42 =

Mk 6, 17-29 =

193 Johannes Ent-

hauptung [29.8.]

194 Maria Geburt

[8.9.]

195 Kreuzerhőhung - 5

[14.9.]

196 Matthăus [21.9.]

Mt 1, 1-16=

197 Michael [29.9.]

Mt 24, 27-35=

Mt 18, 1-10 =

- PPaulus Bekehrung [25.1.]; auch Benedikt [21.3.]\}

- Vgl. ST. Bersser (wie Anm. 6), S. 184 u. S. 179: Oktavtag Petrus u. Paulus Mt 14, $22 f$.

so \{Donnerstag n. 5. Fastensonntag\} Vgl. 80.

si \{Vinzenz [22.1.]\}

52 Vgl. ST. Beussel (wie Anm. 6), S. 186 u. S. 180: Kreuzerhöhung Mt 24, $27 \mathrm{f}$. 


\begin{tabular}{|c|c|c|c|c|c|}
\hline Nr. & Tag in Hs. & $\mathbf{F r}$ & $\mathbf{B r}$ & N1 & $\mathbf{N 2}$ \\
\hline 198 & $\begin{array}{l}\text { Andreas } \\
\text { Vortag [29.11.] }\end{array}$ & Jo $1,35-51$ & 0 & $\circ$ & $=$ \\
\hline 199 & Andreas [30.11.] & Mt 4, 18-22 & $=$ & $=53$ & $=$ \\
\hline
\end{tabular}

Commune

\begin{tabular}{|c|c|c|c|c|c|c|}
\hline 200 & Apostel Vortag & (A) & Jo $15,17-25$ & $\circ$ & 0 & $=$ \\
\hline 201 & Apostel & (A) & Jo $15,12-16$ & $=$ & 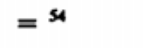 & $=$ \\
\hline 202 & [Apostel] & $\begin{array}{l}\text { Gervasius u. Prota- } \\
\text { sius (A) [19.6.] }\end{array}$ & Mt 10, 16-22 & $=$ & ० & $=$ \\
\hline 203 & [Apostel] & - & $\begin{array}{l}\text { Mk 3, 13-19 } \\
\text { (vgl. 31: Mk } \\
3,6-15)\end{array}$ & $\begin{array}{l}= \\
\text { (vgl. 31) }\end{array}$ & $\begin{array}{l}= \\
\text { (vgl. 31) }\end{array}$ & $\begin{array}{l}= \\
\text { (vgl. 31) }\end{array}$ \\
\hline 204 & Märtyrer & $\begin{array}{l}\text { Felix }(\mathrm{N}) \\
{[14.1 .]}\end{array}$ & Lk 10, 16-20 & $\begin{array}{l}= \\
0 \$ 6\end{array}$ & $\begin{array}{l}=57 \\
056\end{array}$ & $=$ \\
\hline 205 & [Märtyrer] & $\begin{array}{l}\text { Vinzenz }(N) \\
{[22.1 .]}\end{array}$ & Mt 16, 24-28 & $=$ & $=s$ & $=$ \\
\hline 206 & [Märtyrer] & $\begin{array}{l}\text { Christophorus } \\
\text { [25.7.] }\end{array}$ & Lk 14, 26-33 & 0 & 0 & $=$ \\
\hline 207 & Markus [25.4.] & $\begin{array}{l}\text { Bonifatius (A) } \\
\text { [5.6.] }\end{array}$ & Lk 21, 9-19 & $=$ & $\begin{array}{l}=\infty \\
\text { [Märtyrer] }\end{array}$ & $\begin{array}{l}= \\
\text { Märtyrer }\end{array}$ \\
\hline 208 & [Märtyrer] & Bekenner (A) ${ }^{61}$ & Lk 12, 35-40 & $=$ & $=$ & $=$ \\
\hline
\end{tabular}

so \{Hadrian [8.9.]\}

44 \{Vitus [15.6.]; Valentin [14.2.]; Blasius [3.2.]; Simon und Judas [28.10.]\}

ss (N): Bonifatius [5.6.].

\$ Nur Vermerk: ein weiteres Evangelium.

57 \{Märtyrer; Valentin ? [14.2.]\}

se \{Vortag von Laurentius [9.8.]; Wenzel [28.9.]\}

9 Vgl. P. PIETSCH (wie Anm. 1), S. 75: Bonifatius (A) [5.6.]; Marcellinus u. Petrus (N) [2.6.].

๑) \{Märtyrer; Georg [23.4.]\}

61 (N): Benedikt [21.3.]. 


\begin{tabular}{|c|c|c|c|c|c|c|}
\hline Nr. & Tag in Hs. & Normtag & $\mathbf{F r}$ & $\mathbf{B r}$ & N1 & N2 \\
\hline 209 & [Märtyrer] & $\begin{array}{l}\text { Cyriacus mit Ge- } \\
\text { fâhrten (A) [8.8.] }\end{array}$ & Mt 10, 26-32 & $=$ & $\begin{array}{l}= \\
0\end{array}$ & $=$ \\
\hline 210 & [Märtyrer] & $\begin{array}{l}\text { Felix u. Audactus } \\
\text { [30.8.] }\end{array}$ & Mt 24, 3-13 & $\circ$ & $=$ & $=$ \\
\hline 211 & [Mārtyrer] & $-\infty$ & Mt 19, 3-11 & $=$ & $=$ & $=$ \\
\hline 212 & [Märtyrer] & $\begin{array}{l}\text { Gervasius u. Pro- } \\
\text { tasius (N) [19.6.] }\end{array}$ & Mk 13, 1-13 & $=$ & $\circ$ & $=$ \\
\hline 213 & [Märtyrer] & $\begin{array}{l}\text { Fabian u. Sebasti- } \\
\text { an [20.1.] }\end{array}$ & Lk 6, 17-23 & $\circ$ & $\circ$ & $=$ \\
\hline 214 & $\begin{array}{l}\text { Allerheiligen } \\
\text { [1.11.] }\end{array}$ & & Mt 5, 1-12 & $=$ & $=\Phi$ & $=$ \\
\hline 215 & Bischőfe & $\begin{array}{l}\text { Silvester }(\mathrm{N}) \\
\text { [31.12.] }\end{array}$ & Mt 24, 42-47 & $=$ & $\begin{array}{l}=64 \\
056\end{array}$ & $=$ \\
\hline 216 & [Bischōfe] & $\begin{array}{l}\text { Thomas v. Canter- } \\
\text { bury (A) [29.12.] }\end{array}$ & Mt 25, 14-23 & $=$ & $\circ$ & $=$ \\
\hline 217 & [Bischōfe] & Urban (A) [25.5.] & Mk 13, 33-37 & $\begin{array}{l}= \\
0\end{array}$ & $=$ & $=$ \\
\hline 218 & $\begin{array}{l}\text { [Bischöfe] / } \\
\text { Märtyrer }\end{array}$ & $\begin{array}{l}\text { Antonius (A) } \\
{[17.1 .]^{66}}\end{array}$ & $\begin{array}{l}\text { Lk 11, 33-35 } \\
\text { (in Hs noch- } \\
\text { mals nach 94: } \\
\text { Martyrer) }\end{array}$ & $=$ & $=\sigma$ & $=$ \\
\hline 219 & [Bischōfe] & Barnabas [11.6.] & Lk 10, 1-7 & $\begin{array}{l}= \\
\text { (vgl. 225) }\end{array}$ & $=\varpi$ & $=$ \\
\hline 220 & Jungfrauen & Barbara [4.12.] & Mt 25, 1-13 & $=$ & $=\theta$ & $\begin{array}{l}= \\
0 \times\end{array}$ \\
\hline
\end{tabular}

- Vgl. ST. Beissel (wie Anm. 6), S. 136: Nereus u. Achilleus [12.5.].

a \{Auch Elftausend Jungfrauen [21.10.]\}

a \{Bischöfe; Silvester [31.12.]; Urban [25.5.]\}

os (N): Nikolaus [6.12.].

* (N): Vinzenz Abt [11.3.].

ต \{Hieronymus [30.9.]\}

- \{Lukas [18.10.]\}

- \{Margaretha [13.7.]; Luzia [13.12.]\} 


\begin{tabular}{lllllll} 
Nr. & Tag in Hs. & Normtag & Fr & Br & N1 & N2 \\
\hline 221 & [Jungfrauen] & (A) ${ }^{x}$ & Mt 13, 44-52 $=$ & $=n$ & $\begin{array}{l}= \\
0\end{array}$ \\
\hline 222 & Kirchweihe & (N) & Lk 19, 1-10 & $\begin{array}{l}=(3-10 \text { fehlt }) \\
\circ\end{array}$ & $=$ & $=$ \\
\hline 223 & [Kirchweihe] & $-n$ & Lk 6, 43-48 & 0 & $=$ & $=$ \\
\hline
\end{tabular}

\section{Nachtraige in $\mathbf{B r}$}

\begin{tabular}{|c|c|c|c|c|c|}
\hline 224 & - & - & $\circ$ & $\begin{array}{l}\text { Mt 19, 23-26 } \\
\text { (in Hs als Nach- } \\
\text { trag nach 93) }\end{array}$ & $\circ$ \\
\hline (65) & & & & (Mt 15, 1-20) & \\
\hline (4) & & & & $(L k 1,26-38)$ & \\
\hline (172) & & & & $(M t 9,1-8)$ & \\
\hline 225 & - & - & $\circ$ & $\begin{array}{l}\text { Mt 10,1; Lk 9,2-3; } \\
\text { Mt 10,5-10; Lk 10,4 }\end{array}$ & $\circ$ \\
\hline (182) & & & & $(L k 12,13-21)$ & \\
\hline
\end{tabular}

Nachtrag in N2

\begin{tabular}{lllll}
\hline 226 & $\begin{array}{l}\text { Montag } n . \\
\text { Palmsonntag }\end{array}$ & (A) & $\circ$ & $\begin{array}{l}\text { Jo 12, 1-18 } \\
\text { (vgl. 85) }\end{array}$ \\
\hline
\end{tabular}

(N): Luzia [13.12.].

7 Agnes [21.1.]; Elisabeth [19.11.]\}

$n$ Vgl. ST. BelsSEL (wie Anm. 6), S.153: Kirchweihe L 6,43f.

n \{Thomas [21.12.] und Verweis auf 1. Sonntag n. Ostern\} Dieser gesamte Eintrag getilgt. 
Vergleicht man die Anordnung in Fr mit denen anderer hochdeutscher Plenarien, so ist festzustellen, daß die Tagesbezeichnungen vielfach wie auch bisweilen dort nicht mit den Perikopentexten übereinstimmen. Die Reihenfolge der Evangelienabschnitte ist aber im Temporale im großen und ganzen dieselbe, zumindest was die Sonntage betrifft. Die folgende Tabelle zum Temporale läßt Gemeinsamkeiten und Unterschiede deutlich erkennen. Dabei ist bei Übereinstimmung mit der durch die entsprechende Nummer bezeichneten Perikope in Fr ein Gleichheitszeichen, bei Fehlanzeige $\circ$ gesetzt. Bei größeren Abweichungen hinsichtlich des Umfangs des entsprechenden Evangelienabschnitts ist dies vermerkt. Ist eine Perikope verschoben, so ist das Gleichheitszeichen in runde Klammern eingeschlossen und die Stelle, bezogen auf Fr, in einer Anmerkung angeführt. In den Spalten zwei bis fünf sind Bestand und Reihenfolge von vier Plenarien abzulesen. Da es hier nur darum geht, für das Bremer Evangelistar eine breitere Vergleichsgrundlage zur Beurteilung seiner Perikopenanordnung zu geben, ist der überschießende Bestand dieser Plenarien hier nicht vermerkt. Es handelt sich bei drei von ihnen um gedruckt vorliegende Texte: Das Berliner Evangelistar $\mathrm{I}^{\mathbf{4}}$, vertreten durch Ms. germ. $4^{\circ} 533$ der Staatsbibliothek zu Berlin - PreuBischer Kulturbesitz (a.1340, thüringisch-obersächsisch), die Eifler Plenarhandschrift ${ }^{75}$ im Cod. $810 /^{2}$ $13384^{\circ}$ der Trierer Stadtbibliothek (a.1464, moselfränkisch) und die Evangelien der guten Meister von Prag76, vertreten durch den Cod. Lit. 146 der Bamberger Staatsbibliothek (um 1477, mitteldeutsch / oberdeutsch ${ }^{7}$ ). In der letzten Spalte ist die bisher noch nicht edierte Donaueschinger Handschrift Ms. 204 aus der Fürstlich-Fürstenbergschen Hofbibliothek (2. Hälfte 15 . Jh. $^{78}$, bairisch) ausgewertet, die zum Übersetzungszweig der Olmützer Perikopen gehört $^{79}$.

74 GỐNTER FeUder, Das Evangelistar der Berliner Handschrift Ms. germ. 4 533, 2 Teile, Berlin 1961. J. SPLETT (wie Anm. 1), S. 25*.

75 H. Jeske (wie Anm. 12), S. 45ff. J. Splett (wie Anm. 1), S. 40*.

76 Joser WerLIN, Die Evangelien der guten Meister von Prag, Gräfelfing bei München 1962, S.48ff.; ChrISTOPH GERHARDT, Die Evangelien der guten Meister von Prag, München 1970, S. $253 \mathrm{ff}$.; J. SPLETT (wie Anm. 1), S. 34*.

77 J. WerLIN (wie Anm. 76), S. 47: "Sprachlich zeigt der Evangelientext die für die Bamberger Gegend charakteristischen Mischformen aus bayrischen, fränkischen, mitteldeutschen und ostmitteldeutschen Mundarten".

78 Frengut LOSER / Christine STŌLlinger-Lőser, Verteidigung der Laienbibel. In: Überlieferungsgeschichtliche Editionen, Tübingen 1989, S. 266.

79 J. Splett (wie Anm. 1), S.19*ff. Eine Edition der Handschrift wird z. Zt. von ANDreas Kerstan vorbereitet. 
Fr BeE EiP EPr OlP

\begin{tabular}{|c|c|c|c|c|}
\hline $1-36 / 8$ & $=$ & $=$ & $=$ & $=$ \\
\hline 9 & 0 & $(=)^{80}$ & $(=)^{80}$ & $(=)^{80}$ \\
\hline $10-1719$ & $=$ & $=$ & $=$ & $=$ \\
\hline 22 & $(=)^{81}$ & $(=)^{82}$ & $(=)^{81}$ & 0 \\
\hline 25 & $=$ & $=$ & $=$ & 0 \\
\hline 26 & $=$ & $=$ & $=$ & $=$ \\
\hline 27 & $=$ & o & $=$ & $=$ \\
\hline 28 & 0 & $=$ & 0 & $=$ \\
\hline $29-30$ & $=$ & $=$ & $=$ & $=$ \\
\hline 31 & $=$ & $=$ & 0 & $=$ \\
\hline 32 & $=$ & $=$ & $=$ & $=$ \\
\hline 33 & $(=)^{83}$ & $=$ & o & $=$ \\
\hline 34 & $=$ & $(=)^{84}$ & $=$ & $=$ \\
\hline 35 & $=$ & $(=)^{85}$ & $=$ & $=$ \\
\hline 36 & $=$ & $=$ & $(=)^{86}$ & $=$ \\
\hline 37 & $={ }^{87}$ & $(=)^{88}$ & $(=)^{88}$ & $=$ \\
\hline 39 & $=$ & $=$ & $=$ & $=$ \\
\hline 40 & 0 & 0 & 0 & $=$ \\
\hline 41 & $=$ & $={ }^{89}$ & $=$ & $=$ \\
\hline $42-44 \quad 46-51$ & $=$ & $=$ & $=$ & $=$ \\
\hline 52 & $=$ & $=$ & o & $=$ \\
\hline $53-58$ & $=$ & $=$ & $=$ & $=$ \\
\hline $59^{90}$ & $(=)^{91}$ & $(=)^{91}$ & $(=)^{91}$ & $(=)^{91}$ \\
\hline 60 & $=$ & $=$ & $=$ & $=$ \\
\hline 61 & $=$ & $=$ & $=$ & $=92$ \\
\hline
\end{tabular}

Fr BeE EiP EPr OIP

$$
\begin{aligned}
& \text { 62-65 }==2= \\
& 66(=126)=(=)^{93} \circ= \\
& \text { 67-72 }==2= \\
& 73(=165)(=)^{94}(=)^{94}=(=)^{94} \\
& 74==2= \\
& 75 \circ== \\
& \text { 76-79 }=\text { = = } \\
& 80=00= \\
& 81==2= \\
& 82={ }^{95}== \\
& 83 \circ=0= \\
& 85 \circ[0]^{96}=[0]^{96} \\
& 88=\quad{ }^{97}=\quad={ }^{97} \\
& 89 \circ \circ \circ \circ \circ \\
& 91 \quad(=)^{98} \quad(=)^{98} \quad(=)^{98} \quad(=)^{98} \\
& \text { 92-96 }==\text { = } \\
& 97^{99}(=)^{100}(=)^{100}(=)^{100}(=)^{100} \\
& \text { 98-99 }=\text { = }= \\
& 100(=)^{101}(=)^{101}(=)^{101}(=)^{101} \\
& 101 / 103=\text { = }= \\
& 104 \circ== \\
& 105(=)^{102}(=)^{102}(=)^{102}= \\
& \text { 106-108 }=\text { = = } \\
& 109 \circ== \\
& 110 \circ 000
\end{aligned}
$$

93126.94165.

95 Nur Jo 17, 1-11. 96 Aber vorhandenals SchluBteil der Passion am Palmsonntag.

97 Nur Jo 13, 1-15. 98 98/99.

$99 \mathrm{Vgl}$. Verweis 102 in Fr.

$100101 / 103$. $10188 / 92$.

$102107 / 108$. 
Fr BeE EiP EPr OIP

\begin{tabular}{|c|c|c|c|c|}
\hline 111 & $(=)^{103}$ & $(=)^{103}$ & $(=)^{103}$ & 0 \\
\hline $113-116$ & $=$ & $=$ & $=$ & $=$ \\
\hline 117 & $=$ & $=$ & $(=)^{104}$ & $=$ \\
\hline 119 & $=$ & 0 & $=$ & $=$ \\
\hline $120-124$ & $=$ & $=$ & $=$ & $=$ \\
\hline 125 & $(=)^{105}$ & $(=)^{105}$ & $(=)^{105}$ & $(=)^{105}$ \\
\hline $126(=66)$ & $(=)^{106}$ & $=$ & 0 & $=$ \\
\hline 127 & $=$ & $=$ & $=$ & $=$ \\
\hline 130 & $(=)^{107}$ & $(=)^{108}$ & $(=)^{109}$ & $(=)^{107}$ \\
\hline $131-132$ & $=$ & $=$ & $=$ & $=$ \\
\hline 133 & $=$ & $(=)^{107}$ & 0 & $=$ \\
\hline 134 & $=$ & $(=)^{110}$ & $(=)^{111}$ & $={ }^{112}$ \\
\hline 135 & $=$ & $=$ & $=$ & $=$ \\
\hline 136 & $\circ$ & $(=)^{113}$ & $(=)^{114}$ & $(=)^{115}$ \\
\hline 137 & $=$ & $=$ & o & $=$ \\
\hline $138-139$ & $=$ & $=$ & $=$ & $=$ \\
\hline 140 & 0 & $=$ & $=$ & $=$ \\
\hline 141 & 0 & 0 & 0 & 0 \\
\hline 142 & $=$ & $=$ & $=$ & $=$ \\
\hline 143 & $(=)^{116}$ & $={ }^{117}$ & $={ }^{117}$ & $={ }^{117}$ \\
\hline 144 & 0 & 0 & 0 & $\circ$ \\
\hline 145 & $=$ & $=$ & $=$ & $=$ \\
\hline 146 & ० & 0 & 0 & 0 \\
\hline
\end{tabular}

103 Steht im Sanktorale. 104 115/116.

105 123/124.

10666.

$107 \quad 131 / 132$.

$108 \quad 176 / 177$.

109 132/135.

$110 \quad 168 / 172$.

111 159/161; Mk 11, 11-26.

112 Mk 11, 11-23. 113 137/138 bzw. 138/139.

114 168/172; nur Mt 21, 23-27.

11 Nur Mt 21, 23-27. 116 145/148; Mt 16, 1-12. 117 Mt 16, 1-12.
Fr BeE EiP EPr OIP

$147 \circ(=)^{118}==$

$148==2=$

$149(=)^{119}(=)^{120}(=)^{121}(=)^{121}$

$150(=)^{119}=(=)^{121}(=)^{121}$

$151===$

$152 \circ=00$

153-154 $156==$ =

$157(=)^{122}(=)^{119}==$

$158==(=)^{123}(=)^{124}$

$159==2=$

$160(=)^{125}(=)^{126} \circ \quad(=)^{126}$

$161==$ = $=$

$162(=)^{121}(=)^{122}(=)^{122}(=)^{122}$

$163 \circ \circ \circ \circ$

$164(=)^{127}(=)^{122}(=)^{122}(=)^{122}$

$165==$ = $=$

$166(=)^{128}(=)^{128}(=)^{128}(=)^{128}$

$167==\quad=\quad=^{129}$

$168==$ = $=$

$171=(=)^{130}(=)^{131}=$

$172175-178==$ = $=$

$179 \circ==^{132}=$

$180 \circ(=)^{123}(=)^{133}(=)^{123}$
$118 \quad 138 / 139$.
119 153/154.
$120 \quad 172 / 175$.
$121 \quad 151 / 153$.
$122 \quad 167 / 168$.
$123 \quad 127 / 131$.
124 154/156.
125 Steht im Sanktorale.
126 Steht im Sanktorale (Commune); Lk 19,12-27.
127 165/167.
$1281 / 2$.
129 Nur Lk 14, 1-6.
$13033 / 36$.
$131 \quad 135 / 138$.
132 Mt 9,18-26.
$133 \quad 132 / 135$. 
Am Beginn von Fr und der übrigen Handschriften des Bremer Evangelistars fehlt das Evangelium zum 1. Adventssonntag (Mt 21,1-9). Verluste zu Handschriftenbeginn sind nichts Außergewöhnliches und begegnen hier auch im Berliner Kodex (BeE), der aufgrund von Blattverlusten mitten im ersten Satz der Perikope zum 2. Adventssonntag einsetzt ${ }^{134}$. Die Differenzen zwischen den einzelnen Plenarien beruhen vor allem auf Umstellungen, weniger auf Auslassungen oder Ersetzung durch andere Evangelienabschnitte. Dies betrifft zudem fast ausschließlich die Werktage. Hinsichtlich der Sonntagsperikopen gibt es hier Abweichungen nur beim 5. Sonntag n. Dreikönig (35: Mt 11, 25-30) - Umstellung in EiP -, beim 19. Sonntag n. Dreifaltigkeit (171: Mt 13, 24-30) - Umstellung in EiP und EPr - und beim 25. Sonntag n. Dreifaltigkeit (179: Mt 9, 18-22) - Verweis (Jo 6, 5) auf den 4. Fastensonntag (Jo 6, 1-14) in BeE. Die Differenzen beim Palmsonntag hăngen damit zusammen, daß Zahl, Art und Anordnung der Passionen in der Karwoche durch Einfluß der Leben-JhesuÜberlieferung ${ }^{135}$ in den Handschriften des Bremer Evangelistars uneinheitlich und offensichtlich nur hier in dieser Konstellation überliefert sind. Die Donaueschinger Handschrift (OIP) steht jedenfalls insgesamt hinsichtlich der Perikopenanordnung dem Bremer Evangelistar am nächsten.

$\mathrm{Br}$ weicht im Temporale nur geringfügig von $\mathrm{Fr}$ ab. Das Evangelium zum 1. Sonntag n. Dreikönig (20: Lk 2, 42-52) fehlt in Fr wegen einer Textlücke, möglicherweise auch das zum 1. Sonntag n. Dreikönig (24: Mt 23, 13-23). Ebenso fehlen in Fr die Evangelien zum Mittwoch und Freitag n. 20. Sonntag n. Dreifaltigkeit (173: Lk 14, 12-15 bzw. 174: Mt 13, 31-35), allerdings ohne erkennbaren Grund. Die Perikopen zum Freitag n. 6. und 8. Sonntag n. Dreifaltigkeit (141: Mk 8, 11-26 bzw. 147: Mt 23, 13-23) sowie vom Mittwoch n. 15. Sonntag n. Dreifaltigkeit (162: Mk 9, 17-29) sind weder in $\mathrm{Br}$ noch in N1 überliefert. Außerdem fehlen die Verweise 128 und 155. Das Perikopenregister, das nur in $\mathrm{Br}^{136}$ überliefert ist, läßt dies deutlich erkennen. Zur besseren Übersicht sind in dem folgenden zeilengetreuen Abdruck am linken Rand der Kolumnen anstelle einer Zeilenzählung die Nummern der Perikopenauflistung ${ }^{137}$ angegeben. Die Numerierung im Perikopenverzeichnis bezieht sich auf die rote römische Foliierung ${ }^{138}$, die im großen und ganzen korrekt angegeben ist $^{139}$.

134 Vgl. G. FeUder (wie Anm. 74), 1. Teil, S. XV u. S. 1.

135 Siehe unten den Exkurs: Einwirkung der Leben-Jhesu-Ūberlieferung, S. LIII-LV.

136 Von 6. Hand zweispaltig auf fol.4ra bis fol.6va. Vgl. oben S. XV.

137 Vgl. oben S. XXVff.

$138 \mathrm{Vgl}$. die Angaben in der Handschriftenbeschreibung S. XIVff.

139 Einzelne Nummern sind nicht vollstāndig, da sie nur durch Lōsen des Handschrifteneinbandes hätten gelesen werden können. 
Perikopenverzeichnis Br:

1 iz sullent zeichen gesche(n)

2 da Johannes in herdodis banden lag

3 gaberel der engel ist vz gesant

6 Maria stu(n)t off in den dagen

8 der virde Sondag in dem ad uente $s$ (ecundu)m johannem die juden santen uz ir prist(er)e

9 der fritag jn dem jar des richs tyberij

$10 \mathrm{diz}$ ewan(geliu)m leset man an dem crist abent da vns(er) frauwe sant marie wart gemahelt joseph

11 diz ew(angeliu)m leset $\mathrm{ma(n)}$ an dem cristage der keiser august(us) gebot uB

12 an dem cristage zu der and(er)n messe die hirten sprachen zu einander

13 an dem Cristage zu der fron messe jn dem anbegy(n)ne was

14 daz wort an sant Stephens tag jh(es)us sprach zu der schar der juden

15 an sant johans tag jhesus sprach zu sant peter

16 an der kindelin dag der en gel vnsers h(er)n erschein joseph in dem slaff

17 der jars tag da die achtage ein ende hatten

19 der zwelffte tag da ihesus wart gebom in bethlehe(m) juda

20 der erste sondag nach dem zwelfften tage da ihesus wart zwolff jar alt

22 der mitwoch da ih(es)us v(er)name daz joha(n)nes was gefangen
24 der achzehen dag da hero= des dot gelag

25 der ffritag joh(ann)es sach ihesu(m) komen wu yme

26 der ander sontage nach dem achzehen tage $\mathrm{jz}$ was ein hochzijt gemacht in chana ga $=$ lilee

27 der mitwoch jhesus ging $u B$ in der dogent

28 der ffritag jhesus ging $\mathrm{zu} \quad \mathrm{xvj}$ chafarnaum

29 der iij sondag nach dem xvj xij tage da ihesus ging von dem berge

30 der mitwoch jh(es)us ging in dem lande galylea

31 der fritag die pharisey son= derten sich in der judischeit

32 der iiij sontage nach dem achzehenden tage vnser herre ihesus cristus ging in ein schiff

33 der mitwoch $\mathrm{jh}$ (es)us ging in xviij sins vater lant

34 der ffritag vnser $h(e r)$ re vnd sin jongern die ginge(n) uff dem wege

35 der $\mathrm{v}$ sontag nach dem ach= zehen tage jh(es)us sach $\mathrm{zu}$ sy= nen ju(n)gern vnd sprach

36 der mitwoch man brachte ihesus die kint

37 der ffritag jhesus sprach zt der schar der lude

39 der sondag als die gebu(n)den xx zijt an get jhesus sprach mu sinen ju(n)gern

40 der mitwoch da ihesus fur in eyme schiffe uber mere xvj

xix

$\mathbf{x v}$

xv

xv

xvij

xvij

xviij

xix

xix

$\mathbf{x x}$ 
41 der sontag vor der paffen= fasnacht da ein gro $B$ menige kam $z u$ ihesu

42 der mitwoch jhesus sprach zu sinen jưngern

43 An der paffen fasnacht jhesus nam zu yme zwolff siner jưngern

44 der esche tag jhesus sp(ra)ch zù sinen jùngern

46 der ffritag jhesus sagt sinen ju(n)gem

47 SamBtag da iz spade was

48 der erste sondag in der fasten jh(es)us wart gefurret in die wüst von dem heilgen geist

49 der mandag jhesus saget sinen ju(n)gern so des me(n)schen son $k o(m) m e t$ in siner maiestät

50 der dinstag da ih(es)us was ge gangen zu iherusalem

51 der mitwoch der juden bischoff vnd ir schriber ginge(n) zu ihesu

52 donrstag jhesus sprach $2 u$ den juden vnd $\mathbf{z u}$ den die da glaubten

53 ffritag jz was d(er) jude(n) hochzijt

54 SamBtag Nach den sehs tage(n) nam ih(es)us zu yme petru(m) vnd ja= cobu(m) val johannem

55 der ander sondag in der fasten $\mathrm{jh}$ (es)us ging in daz lant tyri vnd sydonis

56 mandag jh(es)us sp(ra)ch zu der schar der juden

57 dinstag $\mathrm{jh}(\mathrm{es}) \mathrm{us}$ rette $\mathbf{m}$ der schar der juden vnd au sine(n) ju(n)gern

58 mitwoch jh(es)us ging $\mathrm{zu}$ iheru= salem

59 Donrstag jhesus sprach $\mathbf{z u}$ sy= nen ju(n)g(er)n iz was ein richer me(n)sch

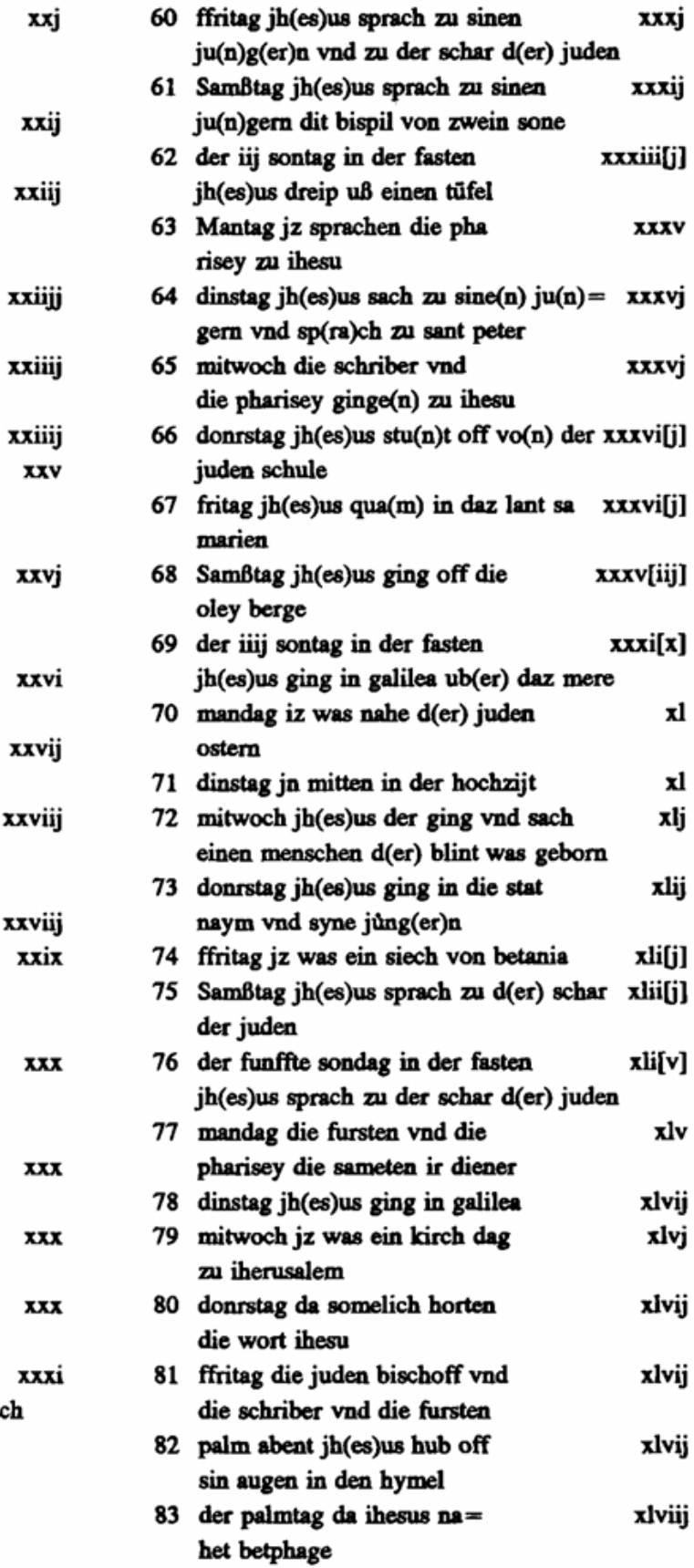

- dawor hanne(m) getilgt 
85 mandag An dem andern tage der da ist nach dem fritage

88 der grüne donrstag vor den heilgen dagen der ostern

90 die passio an dem karfritage jh(es)us ging uber die bach cedron

91 Oster abent Eins samBtages vil frù

$92 \mathrm{diz}$ ewangeliu(m) leset man an dem ostertage vnd beschribet sanct(us) matheus Maria magdalena vnd maria jacobi

93 mantag zwene der jưngern gingen in daz castel

94 dinstag jhesus stunt mitten vnd(er) sinen jongern

95 mitwochen jhesus offente sich bij dem mere tybriadus

96 donrstag maria magdalena stunt bij dem grabe vBerthalb

97 ffritag die wibe gingen uB dem grabe mit fochten

98 SamBtag jn galileam off den berg als in ihesus geboden hatte

99 der erste sondag nach ostern An eime sondag da iz spade wart

100 Ostir abent an dem abent des $\operatorname{sam} B$ tages

101 der mitwoche jhesus erstunt friwe an dem sontage

102 diz ewang(eliu)m vindestu hie vorn der fritag die wibe gingen $u B$ dem grabe mit fochten

103 der ander sondag jhesus sp(ra)ch zu sinen jongern ich bin ein guder hirte

104 mitwochen Eins sondages vil fru

105 ffritag $\mathrm{jh}$ (es)us sprach zu sinen ju(n)= gern der an mich gleubet

106 der dritte sontag nach ostern jhesus sprach mu sinen jungern

107 mitwochen joh(ann)es iz geschach ein frage von s(anc)te joh(ann)es

108 der virde sondag jhesus sprach zu sinen jongern ich gen zu dem

109 mitwochen jh(es)us sp(ra)ch zu sine(n) ju(n)-

$$
\text { Lg(er) } n^{\circ} \quad \text { lviij }
$$

- erstunt] danach an getilgt

- lvj aus lvij korrigiert

- darunter kindelin nachgetragen xlviij

xlix
110 ffritag jhesus sprach m sine(n)

lviij ju(n)gern ich bin ein ware winrebe

111 der funffte sondag jhesus sprach m sinen ju(n)gern uw(er) h(er)cze sal nit druren

112 An des heilgen cruczes tage vnd findet diz ewangelium zu male achtage nach pings = ten jz was ein mensche von den pharisen

113 der funffte sontag jhesus sp(ra)ch b zu sinen jungern jch sagen uch werlich

114 mitwochen wilch evier einerd jhesus sprach m sinen ju(n)g(er)n lx

115 die offart die eilff ju(n)gem saßen bij einander $\mathbf{m l}$ iherusale(m)

117 mitwochen jhesus sprach ma sinen ju(n)gern ich senden uch den

116 lop myns vatir I der sondag nach der offart

119 der pingst abent jh(es)us sprach lxij zu sinen jưngern ist daz ir mich liep hant

120 der pingestag $\mathrm{jh}$ (es)us sprach zu sinen jừngern w(er) mich liep hat

121 mandag $\mathrm{jh}$ (es)us sprach zu sinen jungern also hat got gemy(n)net die werlt

122 dinstag jhesus sprach mu sinen jưngern ich sagen uch werlich

123 mitwochen jh(es)us sprach m sine(n) bxiij jungern iz enmag nyma(n)t $k o(m) m e(n)$

124 donrstag $\mathrm{jz}$ geschach an eim tage daz ihesus saz

125 ffritag jhesus rieff m samen lxxj sinen zwolffbotten

126 SamBtag jhesus stunt off von lxxj der menige der juden

127 der erste sondag nach pingsten von der driualtikeit $j z$ was ein mensche von den phariseyn

130 ffritag jz quam ein richer man

d wilch ... einer nachgetragen

- hie

- I ... offart nachgetragen

Die Zahlenangaben zu den Perikopen 100 - 104 und 110 - 124 aus Korrektur. 
131 der ander sontag jn der zijt seit ihesus sinen jingern

132 der dritte sontag jn der zijt begonden nahen die offen sünder

133 mitwochen jh(es)us sprach ma sine(n) jungern $\mathrm{Si}(\mathrm{n}) \mathrm{t}$ gefolgig uwerm widersachen

134 ffritag jn der zijt ging ih(es)us zu iherusalem

135 der vierde sontag jh(es)us sp(ra)ch zu sinen ju(n)gern sint barmh(er)zig

136 mitwochen da ih(es)us quam in den tempel

137 ffritag jn der zijt fragten ih(es)us jungern

138 der funffte sontag jn der zijt da die menige des folkis

139 der vj sontag jh(es)us sprach zu sinen jungern ich sagen uch

140 mitwoche da ih(es)us ging an dem wege

142 der vij sontag jn der zijt da ein groB schar was mit ihesu

143 mitwochen die saducien vnd die phariseyn

144 ffritag da ihesus ging von dem berge

145 der viii sontag jh(es)us sprach zu sinen jongern hudint uch

146 mitwochen da ihesus gegange(n) was vz dem schiffe an die erden

$148 \mathrm{diz}$ beschribet lucas jh(es)us sagt sinen $\mathrm{ju}(\mathrm{n}) \mathrm{g}(\mathrm{er}) \mathrm{n}$ diz bispil iz waz ein" [rich(er) man in einer stat

149 mitwochen jh(es)us sprach zu sinen jungern so ir sehent iherusalem

150 ffritag $\mathrm{jh}$ (es)us sprach $\mathrm{mu}$ sinen jungern hudent uch

151 der zehende sontag da ih(es)us iherusalem vnd die stat an sach

152 mitwochen jn der zijt da iz abe(n)t was worden

153 der eilffte sontag jhesus sprach zu sinen jungern vnd zu etlich(e)n
Ixxiii

Ixxiiij

lxxiiij

lxxiiij

$\mathbf{x x v}$

$\mathbf{l x x v}$

$\mathbf{l x x v}$

lxxvj

lxxvj

lxuvij

lxxviji

lxxviij

Ixxviij

bxviiji

lxxviiji

$\operatorname{lxxx}$

$\operatorname{lxxx}$

$$
\mathbf{l x x x j}
$$

$\mathbf{l} x \mathbf{x x j}$

$1 \times x \times j$

lxxxij
154 der $x i j$ sontag $\mathrm{jh}(\mathrm{es}) \mathrm{us}$ ging von dem ende tyri

156 der xiij sontag jh(es)us sp(ra)ch w sinen jungern Selig sint die augen

157 mitwoch(e)n da ih(es)us ging durch die stede lerende

158 ffritag $\mathrm{jh}$ (es)us begonde straffen

159 der xiiij sontag da ih(es)us gi(n)g an iherusalem

160 ffritag $j$ h(es)us sprach zu sinen bxxiiij ju(n)gern ez fur ein edelman

161 der xv sontag jh(es)us sprach Du sinen jong(er)n

163 der fritag $j h(e s) u s$ saget di $\chi^{A}$ bispil

164 der sambtag in der fronfasten jh(es)us sagt sinen ju(n)gern diz bij spiel

165 der sechzehen sontag jhesus 1xxxvij ging in die stat naym

166 mitwoch(e)n jh(es)us sprach zu lxxxvi[j] sinen jừngern

167 der xvij sontag jhesus ging an eyme samßtage lxxxv[ij] in eins fursten hus

168 der xviij sontag jz gingen die saducien mu ihesu

169 dise zwey ewangelia findet man in dem aduent ar male der mitwoche Gabrihel der engel ist uB gesant

170 ffritag Maria stu(n)t off in den tage(n) Ixxxix

171 der xix sontag jh(es)us saget sinen ju(n)g(er)n diz bispiel

173 der $\mathbf{x x}$ sontag jh(es)us sprach mil einem fursten der geleret was

174 ffritag $\mathrm{jh}(\mathrm{es}) \mathrm{us}$ sprach m sinen xc jungern

175 der ein vnd zwenzgest sontag jh(es)us sprach $\mathrm{w}$ sinen jongern

176 der $x x i j$ sontag jz was ein konig in kapharnaum

lxxxii[j]

177 der xxiij sontag $\mathrm{jh}(e$ ) us sprach zu sinen jung(er)n diz glichnisse

178 der xxiiij sontag die juden bischoff gingen $\mathrm{m}$ rade

\footnotetext{
- clugen

d die

- das zweite $\mathrm{z}$ abergeschrieben
} Ixxxvij Ixxxviij

lxxxij

lxxij

lxxxiij

lxxxiij

$\operatorname{lxxx[v]}$

$\operatorname{lxx\times v[j]}$

lxxxix

Ixxix

xc

$\mathbf{x c j}$

$\mathbf{x c j}$

xcij
- iz waz ein in der Zeile nachgetragen

b rich(er) ... stat] am linken Rand mit Verweisungszeichen nachgetragen 
179 der xxv sontag da ih(es)us rette mit der menige

180 mitwochen die ju(n)gern gingen zu ihesu vnd sprachen

181 Hie get an die ewangliu(m) von den heiligen jh(es)us sprach zu sine(n) jongern so sie uch forent vor die

183 An sant johans abent baptiste(n) iz was in den tagen

184 an s(anc)te johannes tag baptisten die zijt elizabeth was erfullet

185 an sant peters vnd sant pau= lus abent johannes jhesus sprach zu sinen ju(n)gern

186 an sant peters vnd paul(us) tag jh(es)us quam in daz lant Casarie

188 Sant peters tag $\mathrm{jh}$ (es)us hiez sine jungern in ein schieff gen

189 an sant maria magdalenen tag Ein phariseus bat ihesum daz er mit yme eße

191 an s(anc)te laurencientage jhesus sprach zu sinen jungern

192 Vnser frauwen tag als sie zu himel füre der meist(er) $s p(r i) c h t$ $s$ (anc)te joh(ann)es schribet daz er sach einen starke $n^{\bullet}$ engel

an vnser frauwen tag als sie zu himel füre jh(es)us ging in ein castelle

193 Als sant johans entheubt wart herodes sant boden uz

194 vnser frauwen tag als sie ge= born wart diz ist daz buch des geslechtes

195 des heilgen cruces tag als iz er habin wart $\mathrm{jh}$ (es)us sprach zu sy= nen jongern

196 an sancte matheus tage jh(es)us sach einen menschen siczen an dem zolle

197 An sant michels tage die ju(n)= gern gingen $\mathrm{mu}$ ihesu
199 An sant andreas tage jh(es)us ging bij dem mere

$\mathbf{x c i j}$

xciij

xciji

xciiji

xciiij

xcy

xcv

xcvj

xcvj

xcvij

xcvij

xcviij

xcviij

xcix

xcix

217 S(e)c(un)d(u)m matheum jh(es)us sprach zu sinen jongern wachent 218 Ein ander ewan(geliu)m jh(es)us sprach cvij zu sinen jongern

C

219 Ein ander ewan(geliu)m jh(es)us zeichent cvij auch die and(er)n zwene

$220 \mathrm{an}^{\mathrm{b}}$ der jûngfrauwen tage jhesus seit sinen ju(n)gern diz bispiel

221 Ein ander ew(angeliu)m jh(es)us seit sinen c $j$

cj

cij

cij

cij

ciij

ciij

ciij

ciiij

ciiij

cv

cv

cvj

cvj

cvij 
65 der mitwochen beschribet $\mathrm{ma}=$ theus vnd ist nach dem dritten sontage in der fasten die schri= ber vnd die pharisey gingen zu ihesum

4 lucas ewangelist jn dem sesten mant wart gesant d(er) engel ga= brihel" von got cix

cx

- h durch Uberschreiben aus e gebessert

\section{Exkurs: EINWIRKUNG DER LEBEN-JHESU-ÜBERLIEFERUNG}

Wie der Perikopenauflistung zu entnehmen ist, hat Fr anstelle der über die Karwoche verteilten vier Passionen eine Passionsharmonie am Palmsonntag ${ }^{140}$. Dies verbindet diese Handschrift mit den niederdeutschen Plenarien, in denen - dort allerdings am Karfreitag ebenfalls eine Passie Christi ... vth allen iiij ewangelisten steht ${ }^{141}$. Der in Fr überlieferte Text gehört allerdings zum Übersetzungszweig des Leben Jhesu, der ursprünglich aus dem Mittelniederländischen stammt und sich in hochdeutscher Übersetzung bis ins Alemannische ausgebreitet hat ${ }^{142}$. Bezogen auf die in der Ausgabe von CHRISTOPH GERHARDT berücksichtigten Textzeugen steht er der Hamburger Handschrift U am nächsten ${ }^{143}$. Das gilt noch mehr für die Version, die in der Berliner Handschrift - Staatsbibliothek zu Berlin - Preußischer Kulturbesitz, Ms. germ. qu. 167 - überliefert ist ${ }^{144}$. Deshalb wird in der Edition unter der Nummer 89 diese Handschrift (B) mit den Lesarten von $U$ anstelle der hier ausfallenden drei übrigen Kodizes des Bremer Evangelistars abgedruckt. Hinzuweisen ist in dem Zusammen-

140 Vgl. oben S. XXXI.

141 P. PIETsch (wie Anm. 1), S. 67.

142 Zusammenfassend und mit Literaturhinweisen H. JESKE, 'Evangelienharmonien' I, VL, 2. Auflage, Bd. 2, Berlin 1980, Sp. 646-649.

143 Das Leben Jhesu, Leiden 1970, S.XVf. Vgl. auch WuLYY LÖDTKE, Die Uffenbachsche Evangelien=Harmonie. In: Orientalia Hamburgensia, Hamburg 1926, S. 59-83.

$144 \mathrm{Zu}$ dieser Handschrift vgl. den Hinweis von ERICH ZMMMERMANN, Die deutsche Bibel im religiōsen Leben des Spätmittelalters, BdK. 8, Potsdam 1938, S. 47, und J. SPLETT (Hrsg.), das hymelreich ist gleich einem verporgen schatz in einem acker ..., Gōppingen 1987, S. 24*. 
hang auf ERICH RONNEBURGER ${ }^{145}$, der den Passionstext des Leben Jhesu aus der Münchener Handschrift (M) in Parallele zu dem aus dem Anhang des Evangelienbuches des Matthias von Beheim ${ }^{146}$ abgedruckt hat. Damit ist das Problem der Sonderüberlieferung der Passionsharmonie dieses Übersetzungszweiges und seiner unterschiedlichen Redaktionen angesprochen $^{147}$. Erst wenn dieser Komplex aufgearbeitet ist, wird man möglicherweise genauere Angaben über die Textfassung machen können, die in Fr aus diesem Überlieferungszweig übernommen worden ist.

Auf den Zusammenhang von $\mathrm{Br}$ zur Leben-Jhesu-Überlieferung hatte bereits $\mathrm{W}$. LÜDTKE aufmerksam gemacht ${ }^{148}$. Er wies nach, daB $\mathrm{Br}$ sechs Perikopen bzw. Zusătze aus diesem Übersetzungszweig enthält:

Perikope 4 (Lk 1, 26-38). LJ Kap.3, S.4. Diese Version steht in $\mathrm{Br}$ unter den Nachträgen nach dem Sanktorale auf fol. 119v. Im Temporale folgt Br (fol. 13r) wie auch N1 der Textversion von Fr (Nr. 3), die als Evangelium zum Quatembermittwoch in der Adventszeit vermerkt ist. Unter dem Mittwoch nach dem 18. Sonntag nach Pfingsten (Nr. 169) haben Fr, $\mathrm{Br}$ und N1 jeweils einen Verweis auf diese Perikope (Nr. 3). In N2 steht statt des Verweises die Perikope selbst, aber in einer wiederum anderen Textversion, die hier in der Edition als Nr. 5 eingereiht worden ist.

Perikope 23 (Jo 6, 54-59). LJ Kap.103, S.64, Z.10-20. Diese Perikope, die dem Fronleichnamsfest zuzuordnen ist, ist nur in $\mathrm{Br}$ und dort zweimal überliefert. Auf fol. 20r steht sie vollständig, nochmals - aber in zwei Teilen - von graphischen Varianten und kurzen Auslassungen abgesehen in identischer Form auf fol. $65 \mathrm{v}(6,54)$ und fol. $63 \mathrm{v}(6,55-59)$.

Perikope 65 (Mt 15, 1-20). LJ Kap.105f., S.65, Z.28 - S.67, Z.5. In Br ist diese Perikope zweimal überliefert. Einmal auf fol. 42r für Mittwoch nach dem 3. Fastensonntag, ein zweites Mal auf fol. $118 \mathrm{v}$ in den Nachträgen nach dem Saktorale. Die erste Version orientiert sich an Fr, doch sind hier bereits deutliche Angleichungen an die Leben-Jhesu-Fassung vor-

$145 \mathrm{M}$ [AX] E[DMUND] ERICH RONNEBURGER, Untersuchungen über die deutsche Evangelienharmonie der Münchener Handschrift Cg. 532 aus d. J. 1367, Greifswald 1903, S. 97-119.

146 Leipzig, Universitätsbibliothek, Ms. 34, fol. 224v-234v (E). RONNEBURGER (wie Anm. 145) unter der Sigle L. Vgl. CH. GERHARDT, Das Leben Jhesu. Eine mittelhochdeutsche Evangelienharmonie. Untersuchung, München 1969, S. 39ff.

147 Vgl. auch ROLF KLEMMT, Eine mittelhochdeutsche Evangeliensynopse der Passion Christi. Untersuchung und Text, Leipzig 1964, S. 87ff. Dazu KURT RUH, 'Evangelienharmonien' IV-V. In: VL, 2. Auflage, Bd. 2, Berlin 1980, Sp. 650f.

148 W. LÛDTKE, Evangelientexte, besonders aus Harmonien. In: BdK 11, Potsdam 1941, S. 326f. Die entsprechenden Stellen in der Ausgabe von CH. GERHARDT (wie Anm. 143) werden jeweils unter der Sigle $\mathrm{LJ}$ angegeben. 
genommen worden, die durch die zweite Version vertreten ist. Beide Fassungen in $\mathrm{Br}$ stammen von derselben 5 . Hand.

Perikope 182 (Lk 12, 13-21). LJ Kap.134, S.88. Diese Leben-Jhesu-Version steht in $\mathrm{Br}$ auf fol. 121v als letzter der Nachträge nach dem Sanktorale. Im Perikopenbuch als erstes Evangelium des Sanktorale hat $\mathrm{Br}$ auf fol. $102 \mathrm{r}$ den Text wie auch die Nürnberger Handschriften nach der Version von Fr.

'Perikope' 224 (Mt 19, 23-26). LJ Kap.135, S.89, Z.7-13. Dieser nicht als Perikope belegte Text steht als Nachtrag in $\mathrm{Br}$ auf fol. 65v. Vgl. auch oben zu Perikope 23.

'Perikope' 225 (Mt 10,1; Lc 9, 2-3; Mt 10, 5-10; Lc 10,4). LJ Kap.52, S.31, Z.23 - S.32, Z.11. Dieser synoptische Text steht in $\mathrm{Br}$ auf fol. 121r unter den Nachträgen nach dem Sanktorale. Es bestehen keine Verbindungen zu den Passagen in den Perikopen 125 (Lk 9, 1-6) und 219 (Lk 10, 1-7).

Außer der Perikope 65 haben alle diese Zusätze keine Überschrift, die auf den zugehörigen Tag verweist, sondern nur die jeweiligen Evangelistennamen. Die Texte stehen isoliert vom Perikopenbuch entweder im Nachtrag ab fol. 118v (Perikopen 65, 4, 225, 182) oder nachtragartig auf fol. 20r, 63v, 65v (Perikope 23, 224). Sie stammen alle von der 5. Hand. Mit Ausnahme der nicht als Perikopen bezeugten Texte $(224,225)$ existiert in $\mathrm{Br}$ jeweils eine Parallellversion nach Fr im laufenden Perikopenbuch. Eine Ausnahme bildet die Perikope 23, ebenfalls ohne Überschrift, zu Fronleichnam. Das Evangelium dieses relativ späten Festes $^{149}$ - a.1247 Diözese Lüttich, a.1264 offizielles Fest unter Papst Urban IV, a.1317 allgemeine Verbreitung unter Papst Johannes XXII - ist in den anderen Handschriften nicht überliefert. Möglicherweise sind die angeführten Zusätze in zwei Etappen in die Handschrift eingetragen worden. Denn das Perikopenregister in $\mathrm{Br}$, das von der 6. Hand eingetragen worden ist, verzeichnet nur die Nachträge 65 (fol. 118v) und 4 (fol. 119v), nicht aber die Perikopen 225 und 182 ganz am Ende der Handschrift, auch nicht die innerhalb des Perikopenbuches stehenden Nachträge 23 und $224^{150}$.

Wie Br weicht N1 hinsichtlich Bestand und Anordnung der Perikopen im Temporale nur geringfügig von Fr ab. Hervorzuheben ist, daß das Evangelium zum Sonntag nach Neujahr (18: Lk 2, 33-40) ausschließlich in N1 überliefert ist. Nur in N1 und N2 steht die allgemein zum Oktavtag von Dreikönig gehörende Perikope (21: Mt 3, 13-17). Das gilt auch für die an Maria Lichtmeß (38: Lk 2, 22-32), die allerdings in N1 nochmals im Sanktorale (nach

149 ANGeius A[lbert] Hūussling, 'Fronleichnam'. In: Lexikon des Mittelalters, Bd. 4, München 1989, Sp. 990f. und die dort angegebene Literatur.

150 Vgl. oben S. XIVIII-LIII. 
190) verzeichnet ist. Dagegen fehlen folgende sechs Perikopen nur in N1: 113: Jo 16, 23-30; 114: Lk 11, 5-13; 130: Lk 8, 41-56; 136: Mt 21, 23-32 / 11,5; 146: Lk 8, 27-39; 160: Lk 19, 11-28. In zwei Fällen sind in N1 zwei aufeinanderfolgende Texte lediglich vertauscht: $124 / 125$ und 131/132.

Die Handschrift N2 setzt erst mit dem Evangelium zum Weihnachtsabend (10) ein. Die Perikopen zum Quatembermittwoch und -freitag (5 bzw. 7) stehen an späterer Stelle (vgl. 169 bzw. 170) in jeweils gegenüber Fr anderer Textgestalt. Ausschließlich in N2 sind die drei Passionen nach Matthäus (4), Markus (86) und Lukas (87), das Evangelium zum Freitag nach dem Sonntag nach Christi Himmelfahrt (118: Jo 15, 7-11; vgl. aber 110) und das zum Mittwoch nach dem 1. Sonntag nach Dreifaltigkeit (129: Lk 20, 27-40) überliefert. Wie in N1 sind die Perikopen 124/125 vertauscht. Einmal (165: Lk 7, 11-16) steht statt einer Perikope ein Verweis, und zwar auf 79, wo diese Textstelle in allen vier Handschriften bereits überliefert ist. Andererseits steht in N2 anstelle eines Verweises - so in den anderen Handschriften - der volle Wortlaut noch einmal: 102/97: Mt 28, 8-15. Einmal (112/127) sind in N2 Text und Verweis nur vertauscht.

Das Proprium des Sanktorale verzeichnet keine Heiligen eines speziellen Lokalkalenders, sondern nur allgemein verbreitete Feiertage. Es kann also nicht einer bestimmten Diözese oder einer bestimmten Ordensgemeinschaft zugeordnet werden. Dies gilt ebenfalls für die ins Temporale eingeschobenen Heiligenfeste: Stephanus (26.12.; Perikope 14), Johannes Ev. (27.12.; 15), Unschuldige Kinder (28.12.; 16), Maria LichtmeB (2.2.; 38), Philippus (1.5.; 111) und Kreuzauffindung (3.5.; 112 bzw. 127). Wie die folgende Zusammenstellung zeigt, geben auch die von späterer Hand an den Rändern der Handschrift N1 eingetragenen Perikopenanzeigen keine Hinweise auf einen bestimmten Regionalkalender ${ }^{151}$ :

\begin{tabular}{|c|c|c|}
\hline Agnes (221) & 21.1. & Maria Verkündigung $(169 ; 3)$ \\
\hline Vinzenz (191) & 22.1 . & Tiburtius und Valerianus (110) \\
\hline Paulus (Bekehrung) (187) & 25.1 . & Georg (207) \\
\hline Blasius (201) & 3.2 . & Vortag von Philippus und Ja- \\
\hline Valentin $\left(201 ; 204^{152}\right)$ & 14.2 . & kobus $(110)$ \\
\hline Petrus (Stuhlfeier) (64) & 22.2 . & [Philippus und Jakobus (110) \\
\hline Matthias (35) & 24.2 . & Urban (215) \\
\hline Benedikt (187) & 21.3. & Vitus (201) \\
\hline
\end{tabular}

151 Überprüft anhand der Angaben und Auflistungen bei H. GROTEFEND (wie Anm. 2).

152 Falls das handschriftliche 'sand selden' aus 'sand [f]elden' verschrieben und als Kurzform von Valentin zu fassen ist. 


$\begin{array}{lrlr}\text { Margaretha (220) } & 13.7 . & \text { Elftausend Jungfrauen (214) } & 21.10 . \\ \text { Aussendung der Apostel (115) } & 15.7 . & \text { Simon und Judas (201) } & 28.10 . \\ \text { Vortag von Laurentius (205) } & 9.8 . & \text { Elisabeth (221) } & 19.11 . \\ \text { Hadrian (199) } & 8.9 . & \text { Katharina (25) } & 25.11 . \\ \text { Wenzel (205) } & 28.9 . & \text { Luzia (220) } & 13.12 . \\ \text { Hieronymus (218) } & 30.9 . & \text { [Thomas (220) } & 21.12 .] \\ \text { Lukas (219) } & 18.10 . & \text { Silvester (215) } & 31.12 .\end{array}$

Hinsichtlich Handschrift N2 ist in diesem Zusammenhang nur anzumerken, daB im Temporale die Perikope zum Freitag nach dem 4. Sonntag nach Ostern (110) als die zum Fest von Tiburtius und Valerianus (14.4.) bezeichnet wird, wie dies auch in N1 im Nachtrag von späterer Hand geschehen ist. Am Mittwoch nach dem 5. Sonntag nach Ostern (114) wird die Perikope als die zum Markusfest (25.4.) ausgewiesen. Im Proprium des Sanktorale (185) ist der Vortag (28.6.) fälschlich als Festtag von Peter und Paul bezeichnet. 